\title{
Enhanced ongoing endogenous activity predicts elimination of adult- born neurons in the mouse olfactory bulb
}

Xin Su, Yury Kovalchuk, Nima Mojtahedi \& Olga Garaschuk*

Institute of Physiology, Department of Neurophysiology, Eberhard Karls University of

Tübingen, Tübingen, Germany

${ }^{*}$ Corresponding author

Correspondence should be addressed to:

Prof. Dr. Olga Garaschuk

Department of Neurophysiology, University of Tübingen

Keplerstr. 15, 72074 Tübingen, Germany

Tel: +49-070712973640

Fax: +49-07071295395

E-mail: olga.garaschuk@uni-tuebingen.de 


\section{Abstract}

Adult-born cells, arriving daily into the rodent olfactory bulb, either integrate into the neural circuitry or get eliminated. Whether these two populations differ in their morphological or functional properties remains, however, unclear. Using in vivo twophoton imaging, we monitored longitudinally the dendritic morphogenesis, odor-evoked responsiveness, endogenous $\mathrm{Ca}^{2+}$ signaling and survival/death of adult-born juxtaglomerular neurons (JGNs). We found that JGN maturation is accompanied by a significant reduction in dendritic complexity, with surviving and subsequently eliminated cells showing similar degrees of reduction and dendritic remodeling. Moreover, $\sim 63 \%$ of subsequently eliminated adult-born JGNs acquired odor-responsiveness before death, with amplitudes and time courses of odor-evoked responses similar to those recorded in the surviving cells. We observed, however, a significant long-lasting enhancement of the endogenous $\mathrm{Ca}^{2+}$ signaling in subsequently eliminated JGNs, visible already 6 days before death. These findings identify the ongoing endogenous $\mathrm{Ca}^{2+}$ signaling as a key predictor of the adult-born JGN's fate.

\section{Keywords}

Adult neurogenesis, adult-born cells, two-photon in vivo calcium imaging, survival, death, dendritic morphology, odor-evoked response, endogenous activity. 


\section{Introduction}

Adult neurogenesis provides the brains of grown-ups with an additional level of plasticity and cognitive flexibility ${ }^{1,2}$, which is important for odor-dependent learning, memory encoding, spatial, temporal or odor pattern separation and emotional contro| ${ }^{3,4}$. Altered adult neurogenesis has been associated with several neurodegenerative (e.g. Parkinson's, Alzheimer's and Huntington's disease) and neuropsychiatric disorders, including major depressive disorder, epilepsy, schizophrenia and anxiety ${ }^{5-7}$.

Under physiological conditions, two regions of the adult rodent brain continuously generate new neurons: the subgranular zone of the dentate gyrus, generating hippocampal granule cells, and the subventricular zone (SVZ) of the lateral ventricle, generating adult-born cells $(A B C s)$ of the olfactory bulb $(\mathrm{OB})^{1}$. Neuronal progenitors generated in the SVZ migrate through the rostral migratory stream (RMS) into the OB, where they differentiate into inhibitory interneurons. Approximately $90 \%$ of $A B C s$ become granule cells (GCs), whereas another $5-10 \%$ become juxtaglomerular neurons $(\mathrm{JGNs})^{8,9}$. Based on the pattern of the expressed molecular markers, morphological, electrophysiological and functional properties, adult-born JGNs are further subdivided into two distinct subtypes: adult-born periglomerular cells (PGCs) and short axon cells (SACs) $)^{10,11}$. It takes approximately 4-6 days for ABCs to migrate from the SVZ into the core of the $\mathrm{OB}$, where they start a saltatory (i.e. 'stop-and-go'-like) radial migration ${ }^{12}$. Whereas GCs stop at the end of the radial migration phase, adult-born JGNs speed up and after arrival into the glomerular layer, switch from the radial to the long-distance, unior multi-directional lateral migration, lasting till the end of the pre-integration phase ( 3-4 weeks after $A B C$ 's birth) ${ }^{12}$. 
The patterns of morphogenesis also differ between adult-born GCs and JGNs. Whereas adult-born GCs start to elaborate their dendritic trees after the arrival to the final destination ${ }^{13}$, adult-born JGNs migrate within the glomerular layer with elaborated albeit constantly changing dendritic trees ${ }^{14}$. Longitudinal in vivo imaging showed that $\sim 30$ days after birth, adult-born GCs gain stable dendritic morphology ${ }^{13}$. In adult-born JGNs, so far the development of the dendritic tree was only studied at the population level. The cells increased their complexity between 10 and 45 days after birth, reaching the steady-state thereafter ${ }^{15,16}$. Still, even after maturation, the structural dynamics of dendrites and spines of both adult-born GCs and JGNs remains high, suggesting that $A B C$ s are the most plastic neuronal population in the $\mathrm{OB}^{13,15}$.

Thousands of $A B C$ s migrate into the $O B$ every day but many of them are thought to be eliminated by programmed cell death ${ }^{17-22}$ (but see ref. 23). According to data obtained in bromodeoxyuridine (BrdU) incorporation experiments, elimination of $\mathrm{ABCs}$ mainly happens between 15 and 45 days after birth ${ }^{20-22}$. Plenty of factors, including intracellular signaling molecules and pathways (pro- and anti-apoptotic Bcl-2 family, cAMP response element-binding protein signaling pathway), extracellular signaling molecules (e.g. neurotrophic and growth factors, hormones) and neurotransmitters (GABA, glutamate, serotonin, acetylcholine) were shown to influence the survival of $A B C s^{24-26}$. The survival of $A B C s$ is also modulated by cellular components of the brain's immune system, including microglia and astrocytes 27,28 .

The survival of $A B C s$ is thought to depend on odor sensation, as enriched odor exposure and olfactory discrimination learning promote while odor deprivation (by 
closing one nostril or ablating olfactory sensory neurons) inhibits the survival of $\mathrm{ABCs}^{29-}$ 32. Previous reports suggest the existence of a critical period ( $\sim 2-4$ weeks after birth) when $A B C s$ are most sensitive to the influence of sensory experience ${ }^{31,33,34}$. Since this period corresponds to the pre-integration phase during which $\mathrm{ABC}$ accomplish their migration and start receiving synaptic inputs, it has been postulated that $A B C s$ are eliminated because they "fail to integrate" into the pre-existing neuronal circuitry ${ }^{35,36}$. However, this hypothesis has never been tested directly.

The overall activity level of ABCs is determined by a combination of odor-evoked activity, driven by sensory inputs, and endogenous activity, driven by the spontaneous activity of the surrounding neural network as well as the cell-intrinsic activity. Immature ABCs robustly and broadly respond to odorant stimuli shortly after their arrival into the OB, showing stronger odor-responsiveness but lower odor selectivity compared to mature ABCs or resident cells ${ }^{14,37,38}$. The endogenous activity, represented by $\mathrm{Ca}^{2+}$ spikes, is already present in migrating neuroblasts in the RMS and the subependymal layer of the bulb ${ }^{39,40}$. Spontaneous $\mathrm{Ca}^{2+}$ signals are also ubiquitous in $\mathrm{ABCs}$ which have reached their destination layer ${ }^{41}$. The cell-intrinsic activity has been shown to influence the fate of adult-born GCs, with genetically enhanced cell-intrinsic activity (by expression of the voltage-gated $\mathrm{Na}^{+}$channel $\mathrm{NaChBac}$ ) increasing and genetically suppressed cellintrinsic activity (by expression of Kir2.1 $\mathrm{K}^{+}$channel) decreasing their survival ${ }^{35}$. Knocking out N-methyl-D-aspartate receptors (NMDARs) revealed the critical role of synaptic inputs for the survival of $A B C s^{42,43}$. Noteworthy, the survival of NMDARsdeficient $\mathrm{ABC}$ s was fully rescued by increasing cell-intrinsic activity via $\mathrm{NaChBac}$ 
expression. This suggests that the activity-dependent survival of ABCs depends on the overall level of activity rather than its specific pattern ${ }^{35}$.

It remains, however, unclear whether there are any fundamental morphological or functional differences between the surviving and the subsequently eliminated ABCs. This question can be conveniently addressed in vivo in adult-born JGNs because of their superficial location within the OB. In this study, we employed longitudinal in vivo singlecell tracking and ratiometric two-photon $\mathrm{Ca}^{2+}$ imaging to test whether subsequently eliminated and surviving adult-born JGNs differ in terms of their dendritic morphology, endogenous or sensory-driven $\mathrm{Ca}^{2+}$ signaling. We also examined whether adult-born JGNs are eliminated because they fail to integrate into the pre-existing neural circuitry.

\section{Results}

\section{Longitudinal in vivo mapping of adult-born juxtaglomerular neuron's fate}

For unequivocal identification of migrating adult-born JGNs in longitudinal experiments, we used red-green-blue (RGB) cell-marking approach (Fig. 1a), labeling ABCs via an RMS injection of a mixture of retroviruses encoding mCherry (red), Venus (green) and Cerulean (blue $)^{12}$. This technique provides each labeled cell with a defined color identity, thus enabling accurate tracking of migrating cells over the entire experiment ${ }^{12,44}$. The RGB-labeled adult-born JGNs were visualized through a cranial window in awake mice and their position as well as dendritic morphology were monitored longitudinally starting at 12 days post-viral injection (DPI 12) till DPI 45 (Fig. 1b,c). To develop criteria for the detection of cell elimination, we made use of our prior results showing that cells rarely moved after being stable for more than 4 days (Figure 4 in ref. 12) and left a 30-50- $\mu$ m- 
wide margin to the border of the field of view (FOV), not to mistake cell migration out of the FOV for cell death. Thus, adult-born JGNs were scored as eliminated if (i) cell debris was found in the position where an adult-born JGN was previously located (upper panel in Fig. 1d) or (ii) a cell was present within the FOV at DPI 12, stayed in the same position at DPI 18-22 (at least 4 days) and then disappeared from the dorsal surface of the bulb under the entire cranial window (lower panel in Fig. 1d). At DPI 12-22, cells were scored eliminated only if the cell debris was seen. We often noticed that debris of cells expressing mCherry together with other fluorescent proteins was colored red (e. g. red arrow in Fig. 1d, upper panel), likely due to the higher resistance of mCherry to proteolysis and degradation ${ }^{45}$. Based on the above criteria, RGB-labeled adult-born JGNs were classified as surviving (i.e. staying in the same FOV from DPI 12 to DPI 45), eliminated and uncertain (disappearing from the FOV without fulfilling the above criteria) cells. To avoid false-positive results, we excluded the uncertain cells from further analyses. Note that this might cause an underestimation of the fraction of eliminated cells, reported in this study.

With the above criteria imposed, $61.90 \pm 11.29 \%$ (per mouse) of 528 analyzed adultborn JGNs were classified as surviving cells (Fig. 1e, $\mathrm{n}=12$ mice), $18.45 \pm 12.89 \%$ as eliminated cells (Fig. 1f) and the rest was classified as uncertain cells. The cell turnover within the FOVs was fast during the pre-integration phase ${ }^{12}$ (DPI 12-25) and substantially slower at later time points (Fig. 1g), with the cumulative fraction of eliminated cells increasing almost linearly from DPI 12 to DPI 34 (Fig. 1h; 91 cells, $n=$ 12 mice) and the cumulative fraction of uncertain cells (Fig. 1i) mirroring that of migrating cells in our previous study (see Fig. 7 in ref. 12). In total, $91.21 \%$ of adult-born JGNs 
(83/91 cells) were eliminated between DPI 12 and DPI 28, with no cells eliminated after DPI 34. This data is consistent with the previous in vivo study showing that the elimination of adult-born JGNs after the end of the pre-integration phase is minimal29.

Taken together, our data clearly documented the death of at least $\sim 20 \%$ of adult-born JGNs and also showed that the death was cell age-dependent, mainly happening at early time points. To understand how the decision to die or survive is made, we asked which features of immature adult-born JGNs can predict their future fate.

\section{Development of the dendritic tree in surviving adult-born JGNs}

To begin with, we analyzed the longitudinal change in dendritic morphology. At the population level, it is known that dendritic trees of adult-born JGNs are more complex at DPI 43-45 compared to DPI 10-13 15,16,38, but longitudinal analyses of dendritic development in individual cells are still lacking. The following parameters were used to quantify the dendritic morphology of adult-born JGNs: total dendritic branch length (TDBL), number of dendrites, number of primary dendrites, number of branch points and number of dendritic endings (Fig. 2a). Based on the number of primary dendrites, adultborn JGNs were classified as either unipolar or multipolar cells (Fig. 2b). We found a significant increase in the fraction of unipolar adult-born JGNs from DPI 13 to DPI 45 (Fig. 2c, $P=4.9^{*} 10^{-3}$, Wilcoxon signed rank test, $\mathrm{n}=11$ mice). There are two possible explanations for this result: (i) either multipolar cells pruned their primary dendrites to become unipolar or (ii) the unipolarity of dendritic tree is beneficial for cell survival and more multipolar cells were eliminated at DPI 13-45. Out of all adult-born JGNs with documented dendritic morphology, 22.5\% (9/40 cells, $n=11$ mice) of eliminated and 
$12.16 \%$ of surviving cells ( $18 / 148$ cells, $n=12$ mice) had a unipolar morphology at DPI 13. The observed difference, however, did not reach the level of statistical significance (Fig. 2d, $P=0.16$, Chi-square test with Yates' correction), suggesting that the unipolarity of dendritic tree is not beneficial for the survival of adult-born JGNs. These data also suggested that the higher fraction of unipolar cells observed at DPI 45 (Fig. 2c) was mostly caused by pruning of the primary dendrites of multipolar cells during development. We first analyzed the dendritic morphology of surviving cells that did not change their position between DPI 13 and DPI 45 (called stable surviving ABCs; $69.62 \pm 12.12 \%$ of all surviving cells, $\mathrm{n}=12$ mice) and then took these data as a reference to assess the morphological features of eliminated $A B C s$, which might predict their fate. To do so, we reconstructed and analyzed the dendritic trees of stable surviving $\mathrm{ABC}$ at different time points as indicated in Fig. 2e. For each cell, the data were normalized to the respective value measured at DPI 13 and means of 2 neighboring time points were taken for statistics shown in Fig. 2f-j.

Unexpectedly, all morphological parameters of stable surviving adult-born JGNs including the median (per mouse) TDBL, number of dendrites, number of primary dendrites, number of branch points and number of endings declined with cell's maturation (Fig. 2f-j, $n=34$ cells, 8 mice). The reduction in the number of primary dendrites is consistent with the increased fraction of unipolar cells seen at DPI 45 (Fig. 2c). Concordantly, Sholl analysis also revealed a significant reduction of the dendritic arbor complexity at DPI 45 compared to DPI 13 (Fig. 2k,l). 
To investigate the turnover of dendritic branches, we conducted quantitative 4dimensional structural plasticity analyses (4DSPA), using previously published protocols $^{46,47}$. For each neuron, the degree of dendritic remodeling (i.e. the fractions of pruned or added dendritic endings) was analyzed at 6 different time points (DPI 13-14, DPI 17-18, DPI 21-22, DPI 27-28, DPI 34-35 and DPI 44-45; Fig. 2e). The data showed that stable surviving adult-born JGNs underwent extensive dendritic remodeling at early time points and the degree of structural plasticity decreased over time (Supplementary Fig. 1). Consistent with the literature data ${ }^{15,48}$, even at DPI 44-45, adult-born JGNs remained structurally dynamic (Fig. 2e, Supplementary Fig. 1).

As mentioned above, adult-born JGNs comprise adult-born periglomerular cells (PGCs) and adult-born short axon cells (SACs), which are most probably axonless ${ }^{49}$. These two subtypes have different dendritic morphology. PGCs arborize in a single glomerulus and have short but complex branches, while SACs arborize in several glomeruli and branch less frequently ${ }^{10,50-52}$. Based on their dendritic tree at DPI 45, we classified the adultborn JGNs into PGCs (43.75 $\pm 10.00 \%)$, SACs (46.67 $\pm 20.83 \%)$ and cells (6.25 \pm $18.75 \%$ ) with unclear morphology ( $n=11$ mice). When looking at stable surviving PGCs (Supplementary Fig. 2, $\mathrm{n}=13$ PGCs, 7 mice) and SACs (Supplementary Fig. 3, $\mathrm{n}=14$ SACs, 5 mice), the major aspects of morphological development and dendritic remodeling were similar. Both cell types exhibited a significant reduction in the TDBL as well as the number of primary dendrites from DPI 13 to DPI 45 . For both subtypes, we also observed a decrease in the degree of dendritic remodeling during maturation. Thus, despite the distinct dendritic morphology, adult-born PGCs and SACs share similar patterns of dendritic development. 
In a subset of experiments, we additionally followed the dendritic morphology of adultborn JGNs from DPI 9 to DPI 13. At DPI 9-13, the dendritic morphology of stable surviving adult-born JGNs (i.e. cells, which did not change their position between DPI 13 and DPI 45) was analyzed regardless whether they migrated or not. All the data were normalized to the respective values measured at DPI 13. These data revealed that dendritic trees of stable surviving $A B C$ s grew rapidly at DPI 9-13, increasing, for example, the TDBL by $35.21 \%$ and the number of dendrites by $68.42 \%$ between DPI 9 and DPI 13 (Supplementary Fig. 4, $\mathrm{n}=15$ cells, 4 mice).

Taken together, our data revealed that in stable surviving adult-born JGNs, dendritic growth was restricted to a short time window (DPI 9-13) during the early pre-integration phase. The rest of the maturation time (DPI 13-45) was accompanied by a significant reduction in the size and complexity of the dendritic tree. At early developmental stages, adult-born JGNs underwent both extensive dendritic pruning and addition. The degree of dendritic remodeling decreased during development, with adult-born PGCs and adultborn SACs sharing similar developmental patterns.

\section{Eliminated and surviving adult-born JGNs had a similar level of dendritic complexity and morphological plasticity}

Elimination of immature neurons by apoptosis takes place both during development and in adulthood ${ }^{18,24,53}$. This is a fast process, as the time from the initiation of apoptosis to its completion usually takes less than 24 hours ${ }^{54-57}$. Therefore, to determine the morphological features distinguishing subsequently eliminated from surviving cells, we 
took the day of cell death as a reference point and analyzed the morphology of eliminated adult-born JGNs at one, two and three days before death (DBD 1: 0-24 hours before death, DBD 2: 24-48 hours before death, DBD 3: 48-72 hours before death). The addition and pruning of dendritic endings between two neighboring time points were investigated using the 4DSPA approach. The data obtained were normalized to the respective median values of all (at least 4) surviving cells of the same subtype (i.e. either PGCs-like or SACs-like) recorded in the same mouse at the same time point, as illustrated in Fig. 3a. The terms PGCs-like or SACs-like reflect the fact that the cell's dendritic morphology can be unequivocally determined only in the mature state. Interestingly, even at 1-2 days before death, the subsequently eliminated cells continued to prune and add dendritic endings and we found no significant difference between eliminated and surviving adult-born JGNs for all morphological parameters analyzed, including TDBL (Fig. 3b), number of dendrites (Fig. 3c), number of primary dendrites (Fig. 3d), number of branch points (Fig. 3e), number of endings (Fig. 3f), fraction of pruned (Fig. 3g) and fraction of added (Fig. 3h) endings ( $P>0.05$ for all comparisons, Wilcoxon signed rank test; $\mathrm{n}=10$ eliminated and 63 surviving cells, 6 mice). Thus, our data showed that the complexity of the dendritic tree and the remodeling dynamics cannot predict the subsequent elimination of adult-born JGNs.

\section{Odor-responsiveness as a potential predictor of adult-born JGN's fate}

Since the critical period of adult-born JGN elimination corresponds to the pre-integration phase, when ABCs start to receive synaptic inputs and integrate into the local circuitry, it has been postulated that ABCs die because they "fail to integrate" into the pre-existing neural circuitry ${ }^{35,36,58}$. We took the ability to acquire odor-responsiveness as the readout 
of adult-born JGN's functional integration into the surrounding neural circuitry and tested whether odor-responsiveness protects ABCs from being eliminated. To this end, migrating $A B C s$ in the RMS were labeled by lentivirus encoding the FRET-based $\mathrm{Ca}^{2+}$ indicator Twitch-2B ${ }^{59}$. Because of its ratiometric nature, Twitch-2B is particularly valuable for longitudinal imaging of individual cells in awake animals ${ }^{41,59}$. Since it is difficult to determine the identity of migrating adult-born JGNs without unique color tags, our longitudinal imaging started at DPI 18 (Fig. 4a), when the majority of adult-born JGNs have completed their lateral migration (see Fig. 7A in ref. 12). We focused on the cells which did not change their position during the first three consecutive imaging sessions (DPI 18, 20 and 22), and named them stable adult-born JGNs (Fig. 4b). Focusing on stable, post-migratory $\mathrm{ABC}$ also helped to avoid late-coming cells (yellow arrow in Fig. 4b), arriving in the OB at a low rate after lentiviral injection in the RMS ${ }^{15,37}$. Under these recording conditions, $94.12 \pm 7.10 \%$ (median per mouse) of Twitch-2B+ adult-born JGNs were stable cells (Fig. 4c, $\mathrm{n}=533$ cells, 11 mice). These cells were imaged further every $2^{\text {nd }}$ day in awake mice (Fig. 4a).

Similar to the criteria described for RGB-labeled cells (see above), Twitch-2B+ adult-born JGNs were scored as eliminated either when the cell debris was identified or when stable cells disappeared after DPI 22 (Supplementary Fig. 5). The median (per mouse) fraction of Twitch-2B+ adult-born JGNs eliminated from DPI 22 to DPI 44 was $6.25 \pm$ $5.24 \%(n=11$ mice $)$ and thus similar to the fraction $(5.10 \pm 9.87 \%, n=12$ mice $)$ of $R G B^{+}$ adult-born JGNs eliminated during the same time period (Fig. 4d, $P=0.97$, MannWhitney test). 
Odor-responsiveness of Twitch-2B+ adult-born JGNs was examined by applying the mixture of either 3 or 7 odorants (see Methods) in front of the snout of anesthetized mice. Both kinds of odor mixtures cause broad activation of dorsal glomeruli in the $\mathrm{OB}^{14,38}$, thereby increasing the probability to recruit odor responding adult-born JGNs. Under these experimental conditions, $58.00 \pm 17.44 \%$ (median per mouse, $n=281$ cells tested in 11 mice) of adult-born JGNs showed odor-evoked responses (Fig. 4e-g). Out of 25 subsequently eliminated cells, the odor-evoked responsiveness of 19 cells happened to be tested before they were eliminated. Out of these, $63.16 \%$ of cells $(12 / 19$ cells, 8 mice) showed odor-evoked $\mathrm{Ca}^{2+}$ signals prior to death (Fig. 4h). This convincingly demonstrated that adult-born JGNs can be eliminated despite acquired odorresponsiveness. The mentioned above fraction of odor-responding cells among eliminated adult-born JGNs (63.16\%) was not significantly different from that measured in surviving adult-born JGNs (56.37\%, 146/259 cells, 11 mice) (Fig. 4h, $P=0.74$, Chisquare test with Yates' correction), suggesting that acquired odor-responsiveness per se cannot protect adult-born JGNs from being eliminated.

To investigate whether eliminated and surviving adult-born JGNs responded differently to odor stimulation, we analyzed the maximum $\Delta R / R$ amplitude and the area under the curve (AUC) of odor-evoked $\mathrm{Ca}^{2+}$ transients in eliminated and surviving adult-born JGNs. Then, the median values of eliminated cells were normalized to the median values of corresponding surviving cells recorded from the same mice at the same time points. There was no significant difference between eliminated and surviving cells in terms of the maximum $\Delta \mathrm{R} / \mathrm{R}$ amplitude (Fig. $4 \mathrm{i} ; P=0.49$, Wilcoxon signed rank test) and the AUC (Fig. 4j; $P=0.23$, One sample $t$ test; $\mathrm{n}=12$ odor responding eliminated and 112 
odor responding surviving cells, 8 mice). These data demonstrated the lack of correlations between the properties of odor-evoked responses and the fate of adult-born JGNs.

\section{Distinct endogenous activity levels in eliminated and surviving adult-born JGNs}

Next, we examined whether the Twitch-2B ratio, reflecting cell's intracellular free $\mathrm{Ca}^{2+}$ concentration $\left(\left[\mathrm{Ca}^{2+}\right] \mathrm{i}\right)$, and the pattern of endogenous $\mathrm{Ca}^{2+}$ signaling can predict the fate of adult-born JGNs. To do so, we analyzed endogenous activity patterns of subsequently eliminated cells at DBD 6 (96-144 hours before death), DBD 4 (48-96 hours before death) and DBD 2 (0-48 hours before cell death) and compared them to median activity patterns of all surviving adult-born JGNs recorded in the same mice at the same time points (Fig. 5). Our previous study described the ubiquitous presence of spontaneous $\mathrm{Ca}^{2+}$ transients in the Twitch-2B+ adult-born $\mathrm{JGNs}^{41}$. Using tetrodotoxin, the blocker for voltage-gated $\mathrm{Na}^{+}$channels, we established that Twitch-2 $\mathrm{B}^{+} \mathrm{ABCs}$ can be considered as active (i.e. spiking) when their Twitch-2B ratio is above 2.4 and nonactive (i.e. electrically silent) when their Twitch-2B ratio is below $2.0^{41}$. In the current study, we used this knowledge to analyze the following parameters: basal Twitch-2B ratio, maximum Twitch-2B ratio, maximum $\Delta \mathrm{R} / \mathrm{R}$ amplitude of spontaneous $\mathrm{Ca}^{2+}$ transients, fraction of time spent in the active state (i.e. above $R=2.4$ ), AUC/second (as illustrated in Fig. 5a) and fraction of time spent in the silent state (i.e. below $R=2.0$ ). Many parameters, including the basal (Fig. 5b) and maximum (Fig. 5c) Twitch-2B ratio, fraction of time spent above 2.4 (Fig. 5e), AUC/second (Fig. 5f) and fraction of time spent below 2.0 (except DBD 4; Fig. 5g) were elevated in the subsequently eliminated ABCs $(P<0.05$ for all comparisons, Wilcoxon signed rank test; DBD $6: \mathrm{n}=14$ 
eliminated and 302 surviving cells from 7 mice, DBD 4: 20 eliminated and 466 surviving cells from 8 mice, DBD 2: 16 eliminated and 371 surviving cells from 8 mice). Only the maximum $\Delta R / R$ amplitude (Fig. $5 d$ ) was similar between the subsequently eliminated and surviving $\mathrm{ABCs}$ at all 3 time points.

Next, we investigated the stability of endogenous $\mathrm{Ca}^{2+}$ signaling in surviving and subsequently eliminated adult-born JGNs from DBD 6 to DBD 2. To this end, we took DBD6 as a reference point and calculated, for all parameters under this study, the differences between values measured at DBD 6 and at subsequent time points (Supplementary Fig. 6). Both for the surviving and eliminated cells, the values measured in individual neurons scattered around zero. The median values for surviving cells were remarkably similar in the three recording sessions (Supplementary Fig. $6 ; P>0.05$ for all comparisons, Friedman test). For eliminated adult-born JGNs, however, we did observe a mild increase in the basal (Supplementary Fig. 6a) and maximum (Supplementary Fig. 6b) Twitch-2B ratio and the maximum $\Delta R / R$ amplitude (Supplementary Fig. 6c) at DBD 2 compared to DBD 4 and DBD 6, probably reflecting the apoptosis-related rise of $\left[\mathrm{Ca}^{2+}\right]_{i}^{56,60}$ in some subsequently eliminated cells. Still, we did not detect any significant differences between the surviving and subsequently eliminated ABCs (Supplementary Fig. $6 ; P>0.05$ for all comparisons, Wilcoxon signed rank test; $\mathrm{n}=8$ eliminated cells and 138 surviving cells, 5 mice).

It was suggested that fluctuations of $\left[\mathrm{Ca}^{2+}\right]$ i rather than mere $\mathrm{Ca}^{2+}$ influx are needed to drive the phosphorylation of nuclear cAMP-response-element-binding protein (CREB $)^{61,62}$, a known key mediator of the development and survival of $A B C s^{62-64}$. To 
determine whether adult-born JGNs were eliminated because their $\left[\mathrm{Ca}^{2+}\right]$ i was unable to fluctuate, we analyzed the fluctuations of $\left[\mathrm{Ca}^{2+}\right]$ i both in eliminated and surviving adultborn JGNs (see Methods). At all 3 time points tested (DBD 6, DBD 4, DBD 2), the fractions of cells with fluctuating $\left[\mathrm{Ca}^{2+}\right]_{i}$ did not differ significantly between eliminated and surviving adult-born JGNs (Supplementary Fig. 7; $P>0.05$ for all comparisons, Chisquare test with Yates' correction; DBD 6: $n=14$ eliminated and 302 surviving cells from 7 mice, DBD 4: 20 eliminated and 466 surviving cells from 8 mice, DBD 2: 16 eliminated and 371 surviving cells from 8 mice), suggesting that the high absolute levels of $\left[\mathrm{Ca}^{2+}\right]_{\mathrm{i}}$ rather than the lack of fluctuations were predictive for the subsequent cell death.

Thus, the endogenous activity patterns and the accompanying ongoing changes in $\left[\mathrm{Ca}^{2+}\right]_{i}$ differ significantly between the subsequently eliminated and surviving adult-born JGNs, with subsequently eliminated adult-born JGNs being subjected to increased levels of $\left[\mathrm{Ca}^{2+}\right]$ i over prolonged periods of time.

\section{Discussion}

To our knowledge, this is the first in vivo study analyzing the morphological and functional properties of $\mathrm{ABC}$ prior to their elimination. According to our conservative estimate, subsequently eliminated cells comprise at least some $20 \%$ of adult-born JGNs. The elimination is largely accomplished at the end of the pre-integration phase, with no adult-born JGNs being eliminated after DPI 34. Surprisingly, stable surviving JGNs grew their dendritic trees only during a restricted time period (DPI 9-13) and spent the majority of the maturation time (from DPI 13 onward) reducing and refining their dendritic trees. Despite the distinct morphological appearance, adult-born PGCs and SACs showed 
similar patterns of dendritic development. This knowledge could not have been anticipated based on the previous population-based data, describing the net dendritic growth of adult-born JGNs as the major hallmark of their maturation ${ }^{15,16,38}$.

Despite its complex developmental profile, the morphogenesis occurred similarly in surviving and eliminated JGNs. In addition, we have found that around $63 \%$ of the subsequently eliminated $\mathrm{ABC}$ s acquired odor-responsiveness prior to elimination. This, however, did not prevent their death. Finally, our data clearly showed that the levels of endogenous neuronal activity differ significantly between the surviving and subsequently eliminated adult-born JGNs. Unexpectedly, the death of adult-born JGNs was associated with a protracted increase in the endogenous activity, much in contrast to the previous concept, associating enhanced neuronal activity with increased survival of $\mathrm{ABCs}^{35}$.

It is well established that migrating immature neurons have a highly polarized morphology: a long leading and a short trailing process ${ }^{65,66}$. This is also true for immature $A B C s$ migrating tangentially in the RMS or radially in the deeper layers of the $\mathrm{OB}^{67,68}$. For adult-born GCs, which stop and settle down at the end of radial migration ${ }^{12}$, the steady growth of the dendritic tree ${ }^{13,20}$ is a natural next step in the maturation process. The adult-born JGNs, however, after accomplishing the radial migration enter a 1-3 weeks long lateral migration phase, during which they migrate and simultaneously grow their dendritic trees ${ }^{12,14}$. How these two processes influence each other remains unclear. We showed that adult-born JGNs, which settled down by DPI 13 (irrespective of PGCs or SACs), significantly reduce their dendritic complexity during the subsequent 
development, removing even primary dendrites (Fig. 2). Although surprising and counterintuitive at first glance, these data suggest that while migrating, adult-born JGNs spread their dendrites around to sense activity patterns of many neighboring glomeruli. Once the parent glomeruli ${ }^{14}$ have been found, the cells settle down and start to prune unnecessary dendrites, going beyond their parent glomeruli. This hypothesis can well explain the higher odor-responsiveness but lower odor-selectivity of immature adult-born JGNs, when compared with their mature counterparts ${ }^{38}$. A similar concept might also apply to adult-born GCs. These cells also show overshooting of dendritic complexity, albeit to a much lesser extent ${ }^{13}$, higher odor-responsiveness and lower odor-selectivity ${ }^{37}$ during the maturation process.

The use of a nucleotide analog BrdU as the lineage-tracing tool revealed a significant level of cell death among immature ABCs ( $50 \%$ of cells $)^{20,22,34}$. Recently, an elegant in vivo study from Platel et. al. ${ }^{23}$ has cast doubt on this phenomenon. By using NestinCreER ${ }^{T 2} x$ Rosa-RFP mice, the authors injected tamoxifen to induce RFP expression in SVZ stem cells and all their progeny and measured the number of red cells in the OB once a week between week post-injection 1 and 8 . Analyzed in this way, only $1.5 \%$ of adult-born JGNs and $5.9 \%$ of adult-born GCs disappeared over the observation time period. Similar data were obtained in 48 tdTomato $^{+}$adult-born GCs, labeled by lentiviruses injected into the RMS, prompting the authors to suggest that previously observed cell death is caused by the toxicity of high doses of BrdU23. Although our conservative estimation of the fraction of eliminated adult-born JGNs ( $20 \%)$ is also a factor of 2 lower than that found using BrdU, giving the room to the phenomenon of BrdU-mediated toxicity, it is still 10 times higher than that reported by Platel et. al. The 
explanation is likely to lie in the population-based nature of all previous studies. Indeed, when imaging many (20-170) red cells once a week ${ }^{23}$, it is very difficult to account for vivid cell migration ${ }^{12}$, the substitution of dying cells by late-coming $A B C s^{29}$, etc. Consistently, longitudinal every $2^{\text {nd }}$ day in vivo imaging of adult-born GCs revealed that $\sim 21 \%$ of adult-born GCs were eliminated between DPI 24 and DPI 56 ( 0.7\%/day decline in total cell number $\left.{ }^{13}\right)$. Taken together, these data show that considerable fractions of $\mathrm{ABCs}$ are eliminated after the arrival to their destination layers. For adultborn JGNs, the period of cell elimination seems to start at latest at DPI 12, somewhat earlier than assumed previously ${ }^{23,69}$, and last till DPI 34 only. This time window closely coincides with the period of lateral migration during the pre-integration phase ${ }^{12}$.

Our data show that $\sim 63 \%$ of adult-born JGNs responded to odor stimuli before their death (Fig. 4h). This number is likely underestimated, as instead of determining for each ABC the odorant activating its parent glomerulus ${ }^{14}$, we simply applied a mixture of odorants broadly activating dorsal glomeruli14,38 to increase the throughput. Nonetheless, our data demonstrated that the acquisition of odor-responsiveness does not prevent subsequent cell death. The parameters of odor-evoked responsiveness analyzed in this study (maximum response amplitude and $A \cup C$ ), were also not able to differentiate between surviving and subsequently eliminated cells. We cannot, however, exclude the possibility that other properties (e.g., odor selectivity or the stability of odor responses $^{37,38}$ ) differ in these two groups of cells. For the purpose of this study, we took the cell's odor-responsiveness as a read-out of its functional integration into surrounding neural circuitry. Whether the eliminated odor-responsive cells were indeed synaptically connected, remains unclear. There is a consensus that PSD95-GFP puncta serve as a 
good proxy for putative synapses ${ }^{16,70,71}$. Livneh et. al. have shown that dendrites of adult-born JGNs at DPI 12 are already abundantly decorated by such puncta and the number of puncta increases during the maturation process ${ }^{16}$. Therefore, our eliminated odor-responsive adult-born JGNs, which were older than DPI 22, likely were synaptically connected (i.e., integrated into the pre-existing circuitry) prior to their elimination.

Our data revealed that eliminated adult-born JGNs exhibited elevated endogenous $\mathrm{Ca}^{2+}$ signaling over a prolonged period of time, thus identifying the level of endogenous activity as a potential predictor of cell's fate. This is in strong contrast to the literature data, suggesting that enhanced neuronal activity promotes the survival of immature adult-born $\mathrm{GCs}^{35}$. Although the exact reason for this discrepancy remains to be discovered, it has to be noted that while the $\mathrm{NaChBac}$ expression increased the survival of adult-born GCs in the $\mathrm{OB}^{35}$, it failed to do so in adult-born hippocampal $\mathrm{GCs}^{72}$, suggesting that different types of adult-born cells might possess different survival strategies $^{73}$. In addition, we observed the enhanced endogenous activity in subsequently eliminated ABCs after DPI 18 while in the previous study ${ }^{35}$ the NaChBac expression started at $A B C$ 's birth. Thus, timing may also affect the outcome.

Having discovered that the subsequent death of adult-born JGNs can be predicted by the level of their endogenous $\mathrm{Ca}^{2+}$ signaling, we hypothesized that this is due to the loss of fluctuations in $\left[\mathrm{Ca}^{2+}\right]$, needed for optimal activation of the CREB-mediated survival program $^{61,62}$. Surprisingly, the vast majority of subsequently eliminated adult-born JGNs maintained fluctuations in $\left[\mathrm{Ca}^{2+}\right]_{i}$ even at $\mathrm{DBD} 2$, right before undergoing apoptosis (Supplementary Fig. 7). Although we cannot exclude the possibility that some subtle 
fluctuation features, which are difficult to extract because of the inhomogeneity of the activity patterns in individual Twitch-2B+ cells, do differ between the surviving and the eliminated cells, our current data suggest that the death of adult-born JGNs is triggered by the sustained increase in the overall level of $\left[\mathrm{Ca}^{2+}\right]$ i. In this respect, mitochondria might be an important player. A sustained increase in $\left[\mathrm{Ca}^{2+}\right]$ i causes a sustained increase in the $\mathrm{Ca}^{2+}$ content of the endoplasmic reticulum $(\mathrm{ER})^{74}$ and might promote a direct (via the mitochondrial $\mathrm{Ca}^{2+}$ uniporter or voltage-dependent anion channel) or an ER-mediated (via mitochondria-associated ER membranes) sustained $\mathrm{Ca}^{2+}$ accumulation in the mitochondria 75,76 . The latter can trigger the oxidative stress and the release of proapoptotic factors (e.g. cytochrome c, apoptotic inducing factor, procaspase 9, endonuclease $\mathrm{G}$ ) into the cytosol ${ }^{77,78}$.

Taken together, our study identifies the ongoing endogenous activity as a key player influencing the fate of adult-born JGNs. Such ongoing activity can integrate both the cellintrinsic firing and the influence of the steady-state odor environment reaching either a pro- or an anti-apoptotic level. Because some odorants increase and the others decrease the levels of endogenous activity in $\mathrm{JGNs}^{79}$, this model reconciles the seemingly opposing findings showing that odor enrichment either decreases ${ }^{26}$ or increases $^{80-82}$ the survival of adult-born JGNs.

\section{Materials and methods}

\section{Animals}

All experiments were designed and conducted in accordance with biometrical planning and institutional animal welfare guidelines and were approved by the government of 
Baden-Württemberg, Germany. Three- to four-month-old C57BL/6 mice of either sex were used in this study. Animals were kept in pathogen-free conditions at $22{ }^{\circ} \mathrm{C}$ and $60 \%$ air humidity, under 12 hours light/12 hours dark cycle with ad libitum access to food and water. Female mice were maintained in groups of 3-5 mice, male mice were maintained individually. All experimental procedures were in accordance with the Directive 2010/63/EU of the European Parliament and the Council of the European Union.

\section{Cranial window implantation}

A cranial window above the mouse $O B$ was implanted as described previously ${ }^{12,14,41}$. Briefly, mice were anesthetized by an intraperitoneal (i.p.) injection of ketamine (Fagron, Barsbuettel, Germany) and xylazine (Sigma-Aldrich, MO, USA; 80/4 $\mu \mathrm{g} / \mathrm{g}$ body weight $(B W))$. The depth of anesthesia was estimated using a toe pinch reflex and an additional dose of ketamine/xylazine (40/2 $\mathrm{gg} / \mathrm{g}$ BW) was injected when necessary. Mice were head-fixed in a stereotaxic setup (Stoelting, IL, USA) and a circular groove ( was made above the OB using a high-speed dental drill (ultimate 500, NSK). After careful removal of the bone with fine tweezers (leaving the dura intact), a glass coverslip ( $\varnothing$ $3 \mathrm{~mm}$, Warner Instruments, CT, USA) was gently positioned above the OB. Cyanoacrylate glue was applied to the gap between the coverslip and the skull to stabilize the coverslip, which was further strengthened by blue light-cured dental cement (Ivoclar Vivadent AG, Liechtenstein). After surgery, analgesic carprofen (5 $\mu \mathrm{g} / \mathrm{g} \mathrm{BW}$, Pfizer, Berlin, Germany) was injected subcutaneously for 3 consecutive days and antibiotic enrofloxacin (Baytril, 1:100 v/v, Bayer, Leverkusen, Germany) was applied in drinking water for 10 days. 


\section{Stereotaxic viral injection into the RMS and holder implantation}

Approximately 4 weeks after window implantation, stereotaxic virus injection was performed as described previously ${ }^{12,14,41}$. A glass capillary (tip diameter $\sim 30-40 \mu \mathrm{m}$ ) filled with 1.0-1.5 $\mu$ l of virus-containing solution (composition see below) was navigated to one of the two injection sites at the following coordinates: AP $3.0 \mathrm{~mm}, \mathrm{ML} \pm 0.82 \mathrm{~mm}$, DV $-3.0 \pm 0.05 \mathrm{~mm}$ from pial surface. The viral solution contained either a mixture of retroviruses encoding mCherry, Venus and Cerulean (1:1:1 ratio in terms of infectious titer) ${ }^{12,44}$ or lentivirus encoding the $\mathrm{Ca}^{2+}$ indicator Twitch-2B ${ }^{14,41,59}$. After injection, a custom-made titanium holder was fixed to the skull with blue light-cured dental cement. After surgery, analgesic carprofen (5 $\mu \mathrm{g} / \mathrm{g}$ BW, Pfizer, Berlin, Germany) was injected subcutaneously for 3 consecutive days.

\section{Longitudinal in vivo two-photon imaging}

A training period, allowing the mice to accustom to the experimenter and the setup, started at DPI $5^{41}$. The mice were gently handled inside home cages and then transferred to the microscope stage for 30 minutes of free exploration. At DPI 6-11, after gentle handing and free exploration the mice were head-fixed within the setup for 5 (at DPI 6) to 30-40 (at DPI 11) minutes. Longitudinal in vivo two-photon imaging in awake mice began at DPI 12 .

Image acquisition was performed with a customized two-photon microscope based on an Olympus Fluoview 1000 system (Olympus, Tokyo, Japan), coupled to a mode-locked Ti:Sapphire laser (Mai Tai Deep See, Spectra Physics, CA, USA), as described previously ${ }^{79}$. The emitted fluorescence light was collected through a $20 \mathrm{x}$ water 
immersion objective (NA 1.0, Carl Zeiss, Jena, Germany). Three-dimensional (3D) stacks (512x512 pixels, Kalman filter 2 , step $3 \mu \mathrm{m})$ were acquired daily at DPI 12-35 and DPI 44-45 to document the locations of RGB-positive $\left(\mathrm{RGB}^{+}\right)$cells in awake mice. We used $800 \mathrm{~nm}$ wavelength to excite Cerulean and mCherry and a 570-nm dichroic mirror (570 DM) to split the emission light (the short-pass channel was used for Cerulean and the long-pass channel for mCherry) as well as $970 \mathrm{~nm}$ wavelength to excite Venus (emitted fluorescence was collected via the short-pass channel of 570 DM). Because each labeled cell had its color code, this procedure allowed a precise tracking of individual migrating cells ${ }^{12,44}$. Sparse labeling, blood vessel patterns, tiny irregularities of the dura and the distance between the stable $\mathrm{RGB}^{+}$cell were used as landmarks helping to identify the same cells in longitudinal imaging sessions ${ }^{47}$.

To image the dendritic morphology of $\mathrm{RGB}^{+}$adult-born JGNs, mice were head-fixed under isoflurane anesthesia ( $2 \%$ for induction, $0.8-1.2 \%$ for maintenance) to prevent any animal's movements. Both eyes were protected with eye ointments (Bepanthen, Bayer, Germany). Body temperature was maintained at $36-37^{\circ} \mathrm{C}$ and breath rate was kept at 100-140 breaths per minute (BPM). We chose the excitation wavelength (900 or 970 $\mathrm{nm})$, optimally exciting the expressed fluorescent proteins. The 3D stacks $(512 \times 512$ pixels, Kalman filter 3, step $2 \mu \mathrm{m}$ ) were recorded with appropriate zoom factors (to include the entire dendritic tree).

With the settings optimized for the longitudinal imaging (i.e. high photomultiplier gain, low laser power), we obtained somewhat noisy images (high-noise group, left panel in Supplementary Fig. 8a). To investigate whether this may cause an underestimation of 
the dendritic complexity, we optimized settings for high image quality (i.e. lower photomultiplier gain, higher laser power) and recorded the dendritic morphology of the same adult-born JGNs (low-noise group, right panel in Supplementary Fig. 8a). All the data obtained in the low-noise group were normalized to the respective value measured in the high-noise group (Supplementary Fig. 8b,c). The signal-to-background ratio (SBR) was not significantly different between the 2 groups $(98.47 \pm 39.95 \%, P=0.79$, here and below Wilcoxon signed rank test, $n=12$ cells), but the signal-to-noise ratio (SNR) in the low-noise group showed $\sim 3$ times increase compared to the high-noise group (291.32 \pm $137.63 \%, P<0.001$; Supplementary Fig. 8b). Although the number of dendritic endings was slightly but significantly increased (105.20 $\pm 6.23 \%, P<0.001)$, the improvement of image quality did not cause a substantial increase in the dendritic complexity of adultborn JGNs (TDBL: $101.24 \pm 5.56 \%, P=0.60$, One sample $t$ test; number of dendrites: $101.67 \pm 10.96 \%, P=0.12$, Wilcoxon signed rank test; number of primary dendrites: 100 $\pm 0 \%$; number of branch points: $103.85 \pm 9.76 \%, P=0.08$, One sample $t$ test, $\mathrm{n}=12$ cells; Supplementary Fig. 8c).

\section{In vivo two-photon $\mathrm{Ca}^{2+}$ imaging}

Starting from DPI 11, mice were trained 7 consecutive days for awake imaging, as described above. At DPI 18-44, 3D stacks (512x512 pixels, Kalman filter 2, step $3 \mu \mathrm{m})$ were acquired at $930 \mathrm{~nm}$ excitation wavelength every second day to document the locations of Twitch- $2 \mathrm{~B}^{+}$cells. Endogenous $\mathrm{Ca}^{2+}$ signals were imaged (sampling rate: $6.67 \mathrm{~Hz}$ ) in awake mice at $890 \mathrm{~nm}$ excitation wavelength. Fluorescence signals from mCerulean and $\mathrm{cpVenus}^{\mathrm{CD}}$ were split at $515-\mathrm{nm}$ and filtered with a $475 / 64 \mathrm{~nm}$ band- 
pass filter for mCerulean and a $500 \mathrm{~nm}$ long-pass filter for cpVenus $^{\mathrm{CD}}$ channels, respectively (Semrock, Rochester, United States).

To test whether our longitudinal imaging protocols might exaggerate the elimination of adult-born JGNs, we compared the fraction of stable cells in our RGB and Twitch-2B group to a dataset ( $\mathrm{Li}$ et al., manuscript in preparation), in which Twitch-2B ${ }^{+}$adult-born JGNs were imaged only at 2 time points: either at DPI 14 and 25 or at DPI 25 and 45. We did not detect any significant difference both at DPI 14-25 (Li et al.: $62.50 \pm 23.41 \%$, $\mathrm{n}=5$ mice; RGB group: $61.18 \pm 24.24 \%, \mathrm{n}=12$ mice; $P=0.65$, Mann-Whitney test) and DPI 25-45 (Li et al.: $80.00 \pm 20.76 \%, n=5$ mice; RGB group: $96.29 \pm 7.33 \%, n=12$ mice; Twitch-2B group: $94.74 \pm 4.48 \%, \mathrm{n}=11$ mice; $P=0.07$, Kruskal-Wallis test), suggesting that our longitudinal imaging protocols did not promote the elimination of adult-born JGNs.

\section{Odor application}

Odor-evoked responses were measured as described in ref. 79. In brief, mice were anesthetized with an i.p. injection of midazolam (5 mg/kg BW), medetomidine $(0.5 \mathrm{mg} / \mathrm{kg}$ $\mathrm{BW})$ and fentanyl $(0.05 \mathrm{mg} / \mathrm{kg} \mathrm{BW})$. Body temperature was maintained at $36-37^{\circ} \mathrm{C}$ and breathing rate was 130-170 BPM. A custom-made flow dilution olfactometer was used to deliver a mixture consisting of the equal parts of either 3 (isoamyl acetate, 2-hexanone and 3 ethyl tiglate) or 7 (ethyl-acetate, butanal, pentanal, ethyltiglate, propanal, methylpropionate and ethyl-butyrate) odorants, causing broad activation of dorsal OB glomeruli ${ }^{14,38}$. The mixture of odorants $(1.7 \%$ of saturated vapor) was presented as a 4 second-long pulse in front of the mouse's snout. 


\section{Data analyses}

For the analysis of dendritic morphology, neuronal soma and dendrites were manually traced with Simple Neurite Tracer plugin of Image J (https://imagej.nih.gov/ii). Cells with incomplete dendritic arborizations (e.g. located at the edge of the field of view) were excluded from the analysis. Total dendritic branch length (TDBL), as well as numbers of dendrites, primary dendrites, branch points and endings, were quantified with Neuromantic ${ }^{83}$ and Analyze Skeleton plugin of Image J. We considered dendrites emerging from the soma as primary dendrites, points of dendritic bifurcation as branch points ${ }^{46,84}$, dendrites between the dendrite tip and a branch point ${ }^{47}$ as endings (see also Fig. 2a). Sholl analyses were performed using Simple Neurite Tracer plugin in Image J. Pruned and added dendrites between the 2 consecutive time points were analyzed with the computer-aided 4D structural plasticity analysis (4DSPA) software, according to the established procedure ${ }^{46,47}$. Results were further processed in MATLAB (The MathWorks, Inc., Massachusetts, USA).

For the analysis of SBR and SNR, for each cell 5 dendritic endings were chosen in Fiji. For each dendritic ending, SBR was calculated as the ratio of its mean fluorescent intensity to the mean fluorescent intensity of the corresponding background (darkest area near the cell). SNR was defined as the ratio of ending's mean fluorescent intensity to the standard deviation of the fluorescent intensity of the background. SBR (or SNR) of a cell equaled the median of the SBRs (or SNRs) of its 5 dendritic endings. 
For analyses of the endogenous activity, a somatic region of interest (ROI) and a background $\mathrm{ROI}$ (the darkest area near the target cell) of the comparable size were drawn in Image $\mathrm{J}$. The readouts of mCerulean3 and $\mathrm{cpVenus}^{\mathrm{CD}}$ fluorescence intensity were imported into MATLAB and filtered with a 1st order low-pass digital Butterworth filter with a cutoff frequency of $0.6 \mathrm{~Hz}$. The Twitch-2B ratio was calculated using the following formula:

$$
\text { Ratio }=\frac{F\left(\text { cpVenus }^{\mathrm{CD}}\right) \text { Soma }-\mathrm{F}\left(\text { cpVenus }^{\mathrm{CD}}\right) \text { Background }}{\mathrm{F}(\mathrm{mCerulean} 3) \text { Soma }-\mathrm{F} \text { (mCerulean3) Background }}
$$

The following parameters were analyzed: basal Twitch-2B ratio, maximum Twitch-2B ratio, maximum $\Delta R / R$ amplitude, fraction of time spent above the ratio of 2.4 , area under the curve $(A \cup C)$, fraction of time spent below the ratio of 2.0. Basal and maximum Twitch-2B ratios were calculated as follows: the filtered traces were processed in MATLAB using a sliding average algorithm with a window size of 5 seconds to determine the basal Twitch-2B ratio (minimum average value) and 1.5 seconds to determine the maximum Twitch-2B ratio (maximum average value). The amplitude $(\Delta R / R)$ was calculated as $\left(R-R_{0}\right) / R_{0}$, where $R_{0}$ is the basal Twitch-2B ratio.

In fluctuation analysis, we included the Twitch-2B ratio traces of each eliminated and all (more than 10) corresponding surviving cells, recorded at the same time point from the same mice. In some mice, there were several cells, eliminated at different time points. In this case, we analyzed the Twitch-2B ratio traces of the same surviving adult-born JGNs taken at time points, corresponding to DBD 6, 4 and 2 of the respective eliminated cell. Fluctuations of the Twitch-2B ratio, reflecting fluctuations in the JGN's $\left[\mathrm{Ca}^{2+}\right] \mathrm{i}$, were detected using the mid-reference level crossing approach ${ }^{85}$. The degree of fluctuation 
has been quantified using the number of crossing points passing through the midreference level ${ }^{85}$. The Gaussian mixture model ${ }^{86}$ has been used to detect the naturally existing clusters in the counted crossing points. The correct number of clusters has been estimated using Bayesian Information Criteria ${ }^{87}$. Assigned labels have been used to identify the cells belonging to each cluster among eliminated and corresponding surviving adult-born JGNs. Obtained fractions were compared statistically using the Chisquare test with Yates' correction.

For the analyses of odor-evoked responses, the amplitude was calculated using a custom-written routine in Igor Pro (WaveMetrics, Portland, USA). All $\Delta R / R$ traces were filtered using a binomial filter (time window $0.3 \mathrm{~s}$ ) and the filtered traces were subtracted from the original $\Delta R / R$ traces to obtain the trace of background noise. Cells were defined as odor-responding if the maximum $\Delta R / R$ amplitude of their odor-evoked signals was 3 times larger than the standard deviation of the corresponding background noise.

\section{Statistical analyses}

Unless indicated, all data are shown as median \pm interquartile range (IQR). Statistical analyses were performed using GraphPad Prism 7 (GraphPad Software, CA, USA). Shapiro-Wilk test was used to check the normality of data distribution in individual datasets. For analyses of a single dataset, One sample $t$ test or Wilcoxon signed rank test was applied. For two unpaired datasets, either Unpaired $t$ test or Mann-Whitney test were used, depending on the normality of datasets. For two paired datasets, Paired $t$ test or Wilcoxon signed rank test were applied. For 3 or more paired datasets, Friedman test followed by Dunn's multiple comparisons test or One-way repeated measures 
ANOVA with or without Greenhause-Geisser Sphericity Correction followed by HolmSidak post hoc test was applied. Unless indicated, all statistical tests were two-tailed. Differences were considered significant if $P<0.05$.

\section{Acknowledgments}

We thank E. Zirdum, A. Weible and K. Schoentag for technical assistance, K. Li and K. Figarella for viral production, N. Asavapanumas for help with endogenous activity analysis, A. Gohl, J. Mueck, M. Knecht and S. Wang for help with morphological analysis, Y. Liang and K. Li for critical comments on the manuscript. This work was funded by the Deutsche Forschungsgemeinschaft (GA 654/14-1). X.S. was partially supported by the DAAD Graduate School Scholarship Program (57145465).

\section{Author contributions}

O.G. initiated and conceived the study. X.S. and Y.K. performed experiments. X.S. analyzed the data. N.M. contributed to data analyses. X.S. and O.G. wrote the manuscript. All authors have approved the final version of the manuscript.

\section{Conflict of interest}

The authors declare no conflict of interest. 


\section{References}

1. Ming, G. li \& Song, H. Adult Neurogenesis in the Mammalian Brain: Significant Answers and Significant Questions. Neuron 70, 687-702 (2011).

2. Doetsch, F. \& Hen, R. Young and excitable: The function of new neurons in the adult mammalian brain. Current Opinion in Neurobiology 15, 121-128 (2005).

3. Alonso, M. et al. Activation of adult-born neurons facilitates learning and memory. Nat. Neurosci. 15, 897-904 (2012).

4. Anacker, C. \& Hen, R. Adult hippocampal neurogenesis and cognitive flexibilitylinking memory and mood. Nature Reviews Neuroscience 18, 335-346 (2017).

5. Parent, J. M. \& Murphy, G. G. Mechanisms and functional significance of aberrant seizure-induced hippocampal neurogenesis. Epilepsia 49, 19-25 (2008).

6. Gonçalves, J. T., Schafer, S. T. \& Gage, F. H. Adult Neurogenesis in the Hippocampus: From Stem Cells to Behavior. Cell 167, 897-914 (2016).

7. Braun, S. M. G. \& Jessberger, S. Adult neurogenesis: Mechanisms and functional significance. Dev. 141, 1983-1986 (2014).

8. Bonfanti, L. \& Peretto, P. Adult neurogenesis in mammals - a theme with many variations. Eur. J. Neurosci. 34, 930-950 (2011).

9. Alvarez-Buylla, A. \& García-Verdugo, J. M. Neurogenesis in adult subventricular zone. Journal of Neuroscience 22, 629-634 (2002).

10. Nagayama, S., Homma, R. \& Imamura, F. Neuronal organization of olfactory bulb circuits. Frontiers in Neural Circuits 8, 98 (2014).

11. Kosaka, T. \& Kosaka, K. 'Interneurons' in the olfactory bulb revisited. Neurosci. Res. 69, 93-99 (2011).

12. Liang, Y. et al. Long-term in vivo single-cell tracking reveals the switch of 
migration patterns in adult-born juxtaglomerular cells of the mouse olfactory bulb. Cell Res. 26, 805-821 (2016).

13. Sailor, K. A. et al. Persistent Structural Plasticity Optimizes Sensory Information Processing in the Olfactory Bulb. Neuron 91, 384-396 (2016).

14. Kovalchuk, Y. et al. In vivo odourant response properties of migrating adult-born neurons in the mouse olfactory bulb. Nat. Commun. 6, (2015).

15. Mizrahi, A. Dendritic development and plasticity of adult-born neurons in the mouse olfactory bulb. Nat. Neurosci. 10, 444-452 (2007).

16. Livneh, Y., Feinstein, N., Klein, M. \& Mizrahi, A. Sensory input enhances synaptogenesis of adult-born neurons. J. Neurosci. 29, 86-97 (2009).

17. Biebl, M., Cooper, C. M., Winkler, J. \& Kuhn, H. G. Analysis of neurogenesis and programmed cell death reveals a self- renewing capacity in the adult rat brain. Neurosci. Lett. 291, 17-20 (2000).

18. Biebl, M., Winner, B. \& Winkler, J. Caspase inhibition decreases cell death in regions of adult neurogenesis. Neuroreport 16, 1147-1150 (2005).

19. Woon, R. K. et al. Impaired migration in the rostral migratory stream but spared olfactory function after the elimination of programmed cell death in bax knock-out mice. J. Neurosci. 27, 14392-14403 (2007).

20. Petreanu, L. \& Alvarez-Buylla, A. Maturation and death of adult-born olfactory bulb granule neurons: Role of olfaction. J. Neurosci. 22, 6106-6113 (2002).

21. Whitman, M. C. \& Greer, C. A. Adult-generated neurons exhibit diverse developmental fates. Dev. Neurobiol. 67, 1079-1093 (2007).

22. Winner, B., Cooper-Kuhn, C. M., Aigner, R., Winkler, J. \& Kuhn, H. G. Long-term survival and cell death of newly generated neurons in the adult rat olfactory bulb. 
Eur. J. Neurosci. 16, 1681-1689 (2002).

23. Platel, J. C. et al. Neuronal integration in the adult mouse olfactory bulb is a nonselective addition process. Elife 8, (2019).

24. Kuhn, H. G. Control of cell survival in adult mammalian neurogenesis. Cold Spring Harb. Perspect. Biol. 7, a018895 (2015).

25. Benn, S. C. \& Woolf, C. J. Adult neuron survival strategies - Slamming on the brakes. Nature Reviews Neuroscience 5, 686-700 (2004).

26. Khodosevich, K. et al. Connective Tissue Growth Factor Regulates Interneuron Survival and Information Processing in the Olfactory Bulb. Neuron 79, 1136-1151 (2013).

27. Sultan, S. et al. Synaptic Integration of Adult-Born Hippocampal Neurons Is Locally Controlled by Astrocytes. Neuron 88, 957-972 (2015).

28. Sierra, A. et al. Microglia shape adult hippocampal neurogenesis through apoptosis-coupled phagocytosis. Cell Stem Cell 7, 483-495 (2010).

29. Sawada, M. et al. Sensory input regulates spatial and subtype-specific patterns of neuronal turnover in the adult olfactory bulb. J. Neurosci. 31, 11587-11596 (2011).

30. Sultan, S., Rey, N., Sacquet, J., Mandairon, N. \& Didier, A. Newborn neurons in the olfactory bulb selected for long-term survival through olfactory learning are prematurely suppressed when the olfactory memory is erased. J. Neurosci. $\mathbf{3 1}$, 14893-14898 (2011).

31. Alonso, M. et al. Olfactory discrimination learning increases the survival of adultborn neurons in the olfactory bulb. J. Neurosci. 26, 10508-10513 (2006).

32. Corotto, F. S., Henegar, J. R. \& Maruniak, J. A. Odor deprivation leads to reduced neurogenesis and reduced neuronal survival in the olfactory bulb of the adult 
mouse. Neuroscience 61, 739-744 (1994).

33. Mouret, A. et al. Learning and survival of newly generated neurons: When time matters. J. Neurosci. 28, 11511-11516 (2008).

34. Yamaguchi, M. \& Mori, K. Critical period for sensory experience-dependent survival of newly generated granule cells in the adult mouse olfactory bulb. Proc. Natl. Acad. Sci. U. S. A. 102, 9697-9702 (2005).

35. Lin, C. W. et al. Genetically Increased Cell-Intrinsic Excitability Enhances Neuronal Integration into Adult Brain Circuits. Neuron 65, 32-39 (2010).

36. Turnley, A. M., Basrai, H. S. \& Christie, K. J. Is integration and survival of newborn neurons the bottleneck for effective neural repair by endogenous neural precursor cells? Front. Neurosci. 8, 29 (2014).

37. Wallace, J. L., Wienisch, M. \& Murthy, V. N. Development and Refinement of Functional Properties of Adult-Born Neurons. Neuron 96, 883-896.e7 (2017).

38. Livneh, Y., Adam, Y. \& Mizrahi, A. Odor processing by adult-born neurons. Neuron 81, 1097-1110 (2014).

39. García-González, D. et al. Serotonergic Projections Govern Postnatal Neuroblast Migration. Neuron 94, 534-549 (2017).

40. Darcy, D. P. \& Isaacson, J. S. L-type calcium channels govern calcium signaling in migrating newborn neurons in the postnatal olfactory bulb. J. Neurosci. 29, 2510_ 2518 (2009).

41. Maslyukov, A., Li, K., Su, X., Kovalchuk, Y. \& Garaschuk, O. Spontaneous calcium transients in the immature adult-born neurons of the olfactory bulb. Cell Calcium 74, 43-52 (2018).

42. Tashiro, A., Sandler, V. M., Toni, N., Zhao, C. \& Gage, F. H. NMDA-receptor- 
mediated, cell-specific integration of new neurons in adult dentate gyrus. Nature 442, 929-933 (2006).

43. Kelsch, W., Li, Z., Eliava, M., Goengrich, C. \& Monyer, H. GluN2B-containing NMDA receptors promote wiring of adult-born neurons into olfactory bulb circuits. J. Neurosci. 32, 12603-12611 (2012).

44. Gomez-Nicola, D., Riecken, K., Fehse, B. \& Perry, V. H. In-vivo RGB marking and multicolour single-cell tracking in the adult brain. Sci. Rep. 4, 1-10 (2014).

45. Costantini, L. M. et al. A palette of fluorescent proteins optimized for diverse cellular environments. Nat. Commun. 6, 1-13 (2015).

46. Lee, P. C., He, H. Y., Lin, C. Y., Ching, Y. T. \& Cline, H. T. Computer aided alignment and quantitative 4D structural plasticity analysis of neurons.

Neuroinformatics 11, 249-257 (2013).

47. Gonçalves, J. T. et al. In vivo imaging of dendritic pruning in dentate granule cells. Nat. Neurosci. 19, 788-791 (2016).

48. Livneh, Y. \& Mizrahi, A. Long-term changes in the morphology and synaptic distributions of adult-born neurons. J. Comp. Neurol. 519, 2212-2224 (2011).

49. Galliano, E. et al. Embryonic and postnatal neurogenesis produce functionally distinct subclasses of dopaminergic neuron. Elife 7, (2018).

50. Kiyokage, E. et al. Molecular identity of periglomerular and short axon cells. J. Neurosci. 30, 1185-1196 (2010).

51. Pinching, A. J. \& Powell, T. P. The neuron types of the glomerular layer of the olfactory bulb. J. Cell Sci. 9, 305-345 (1971).

52. Bywalez, W. G., Ona-Jodar, T., Lukas, M., Ninkovic, J. \& Egger, V. Dendritic arborization patterns of small juxtaglomerular cell subtypes within the rodent 
olfactory bulb. Front. Neuroanat. 10, (2017).

53. Yuan, J. \& Yankner, B. A. Apoptosis in the nervous system. Nature 407, 802-809 (2000).

54. Elmore, S. Apoptosis: A Review of Programmed Cell Death. Toxicologic Pathology 35, 495-516 (2007).

55. Saraste, A. Morphologic criteria and detection of apoptosis. Herz 24, 189-195 (1999).

56. Cellerino, A., Galli-Resta, L. \& Colombaioni, L. The dynamics of neuronal death: a time-lapse study in the retina. J. Neurosci. 20, (2000).

57. Cordeiro, M. F. et al. Real-time imaging of single nerve cell apoptosis in retinal neurodegeneration. Proc. Natl. Acad. Sci. U. S. A. 101, 13352-13356 (2004).

58. Lepousez, G. \& Lledo, P.-M. Life and Death Decision in Adult Neurogenesis: In Praise of Napping. Neuron 71, 768-771 (2011).

59. Thestrup, T. et al. Optimized ratiometric calcium sensors for functional in vivo imaging of neurons and T lymphocytes. Nat. Methods 11, 175-182 (2014).

60. Linden, R., Martins, R. A. P. \& Silveira, M. S. Control of programmed cell death by neurotransmitters and neuropeptides in the developing mammalian retina.

Progress in Retinal and Eye Research 24, 457-491 (2005).

61. Li, S., Zhang, C., Takemori, H., Zhou, Y. \& Xiong, Z. Q. TORC1 regulates activitydependent CREB-target gene transcription and dendritic growth of developing cortical neurons. J. Neurosci. 29, 2334-2343 (2009).

62. Li, B., Tadross, M. R. \& Tsien, R. W. Sequential ionic and conformational signaling by calcium channels drives neuronal gene expression. Science (80-. ). 351, 863867 (2016). 
63. Jagasia, R. et al. GABA-cAMP response element-binding protein signaling regulates maturation and survival of newly generated neurons in the adult hippocampus. J. Neurosci. 29, 7966-7977 (2009).

64. Giachino, C. et al. cAMP response element-binding protein regulates differentiation and survival of newborn neurons in the olfactory bulb. J. Neurosci. 25, 10105-10118 (2005).

65. Ota, H. et al. Speed control for neuronal migration in the postnatal brain by Gmipmediated local inactivation of RhoA. Nat. Commun. 5, 1-12 (2014).

66. Buchsbaum, I. Y. \& Cappello, S. Neuronal migration in the CNS during development and disease: Insights from in vivo and in vitro models. Development (Cambridge) 146, (2019).

67. Doetsch, F., García-Verdugo, J. M. \& Alvarez-Buylla, A. Cellular composition and three-dimensional organization of the subventricular germinal zone in the adult mammalian brain. J. Neurosci. 17, 5046-5061 (1997).

68. Kaneko, N., Sawada, M. \& Sawamoto, K. Mechanisms of neuronal migration in the adult brain. J. Neurochem. 141, 835-847 (2017).

69. Mandairon, N., Sacquet, J., Jourdan, F. \& Didier, A. Long-term fate and distribution of newborn cells in the adult mouse olfactory bulb: Influences of olfactory deprivation. Neuroscience 141, 443-451 (2006).

70. Kelsch, W., Lin, C. W. \& Lois, C. Sequential development of synapses in dendritic domains during adult neurogenesis. Proc. Natl. Acad. Sci. U. S. A. 105, 1680316808 (2008).

71. Kelsch, W., Lin, C. W., Mosley, C. P. \& Lois, C. A critical period for activitydependent synaptic development during olfactory bulb adult neurogenesis. J. 
Neurosci. 29, 11852-11858 (2009).

72. Sim, S., Antolin, S., Lin, C. W., Lin, Y. X. \& Lois, C. Increased cell-intrinsic excitability induces synaptic changes in new neurons in the adult dentate gyrus that require Npas4. J. Neurosci. 33, 7928-7940 (2013).

73. Pfisterer, U. \& Khodosevich, K. Neuronal survival in the brain: Neuron typespecific mechanisms. Cell Death and Disease 8, e2643-e2643 (2017).

74. Garaschuk, O., Yaari, Y. \& Konnerth, A. Release and sequestration of calcium by ryanodine-sensitive stores in rat hippocampal neurones. J. Physiol. 502, 13-30 (1997).

75. Celsi, F. et al. Mitochondria, calcium and cell death: A deadly triad in neurodegeneration. Biochimica et Biophysica Acta - Bioenergetics 1787, 335-344 (2009).

76. Rizzuto, R., De Stefani, D., Raffaello, A. \& Mammucari, C. Mitochondria as sensors and regulators of calcium signalling. Nature Reviews Molecular Cell Biology 13, 566-578 (2012).

77. Giorgi, C. et al. Mitochondrial Ca2+ and apoptosis. Cell Calcium 52, 36-43 (2012).

78. Calvo-Rodriguez, M. et al. Increased mitochondrial calcium levels associated with neuronal death in a mouse model of Alzheimer's disease. Nat. Commun. 11, 1-17 (2020).

79. Homma, R., Kovalchuk, Y., Konnerth, A., Cohen, L. B. \& Garaschuk, O. In vivo functional properties of juxtaglomerular neurons in the mouse olfactory bulb. Front. Neural Circuits 7, 23 (2013).

80. Rochefort, C., Gheusi, G., Vincent, J. D. \& Lledo, P. M. Enriched Odor Exposure Increases the Number of Newborn Neurons in the Adult Olfactory Bulb and 
Improves Odor Memory. J. Neurosci. 22, 2679-2689 (2002).

81. Rey, N. L., Sacquet, J., Veyrac, A., Jourdan, F. \& Didier, A. Behavioral and cellular markers of olfactory aging and their response to enrichment. Neurobiol. Aging 33, 626.e9-626.e23 (2012).

82. Forest, J. et al. Short-term availability of adult-born neurons for memory encoding. Nat. Commun. 10, (2019).

83. Myatt, D. R., Hadlington, T., Ascoli, G. A. \& Nasuto, S. J. Neuromantic - from Semi-Manual to Semi-Automatic Reconstruction of Neuron Morphology. Front. Neuroinform. 6, 4 (2012).

84. Ledderose, J., Sención, L., Salgado, H., Arias-Carrión, O. \& Treviño, M. A software tool for the analysis of neuronal morphology data. International Archives of Medicine 7, 1-9 (2014).

85. IEEE Instrumentation and Measurement Society. IEEE Standard for Transitions, Pulses, and Related Waveforms. IEEE-SA Standards Board 2011, (2011).

86. McLachlan, G. J. \& Peel, D. Finite mixture models. (Wiley, 2000).

87. Wit, E., Heuvel, E. van den \& Romeijn, J.-W. 'All models are wrong...': an introduction to model uncertainty. Stat. Neerl. 66, 217-236 (2012). 


\section{Figure legend}

Figure 1. Longitudinal mapping of adult-born JGN's fate. (a) Scheme of the experimental setup. Adult-born JGNs were labeled via RMS injection of a 1:1:1 mixture of RGB retroviruses encoding Venus (green), mCherry (red) and Cerulean (blue) fluorescent proteins and repeatedly imaged via a chronic cranial window by means of two-photon (2P) microscopy. (b) Illustration of the experimental timeline. (c) Maximum intensity projection (MIP) images $(0-100 \mu \mathrm{m}$, step $2 \mu \mathrm{m})$ of RGB ${ }^{+}$adult-born JGNs taken at DPI 12 in red, green and blue channels. The merged image is shown in the lower right corner. Scale bar: $50 \mu \mathrm{m}$. (d) Upper: MIP images $(34-54 \mu \mathrm{m}$, step $2 \mu \mathrm{m}$, $800 \mathrm{~nm}$ excitation wavelength) showing 1 eliminated cell (magenta arrow) and 2 surviving cells (green arrows) in the same FOV at 4 time points: DPI 22 (1 day before death), DPI 23 (the day of death), DPI 24 (1 day after death) and DPI 27 (4 days after death). Note red cell debris visible at DPI 23 and DPI 24. Lower: MIP images (12-52 $\mu \mathrm{m}$, step $2 \mu \mathrm{m}, 900$ $\mathrm{nm}$ excitation wavelength) illustrating 1 eliminated and 1 surviving adult-born JGNs in the same FOV at 5 different time points. The day of death was DPI 29. Scale bars: 25 $\mu \mathrm{m} .(\mathbf{e}, \mathbf{f})$ Box plots showing the median (per mouse) fractions of surviving (e) and eliminated (f) adult-born JGNs at DPI $12-45$ ( $\mathrm{n}=12$ mice). (g) Graph showing the fraction of RGB+ adult-born JGNs present within FOVs at different DPIs. The number of cells present at DPI 12 is taken as $100 \%$. (h,i) Graphs showing cumulative fractions of eliminated (h) and uncertain (i) cells at DPI 12-45 $(n=12$ mice).

Figure 2. Development of the dendritic tree in stable surviving adult-born JGNs. (a) 2D projection image of a reconstructed adult-born JGN (DPI 13) exemplifying the morphological parameters analyzed in this study. Primary dendrites are color-coded red, 
some branch points blue and some dendritic endings cyan. Scale bar: $25 \mu \mathrm{m}$. (b) Representative reconstructions of 2 unipolar and 2 multipolar cells at DPI 45 . Scale bar: $25 \mu \mathrm{m}$. (c) Connected dot graph showing the fractions of unipolar cells at DPI 13 and DPI $45\left(P=4.9^{*} 10^{-3}\right.$, Wilcoxon signed rank test; $n=11$ mice $)$. (d) Bar graph showing the fractions of unipolar and multipolar cells among the eliminated (left) and surviving (right) cells ( $P=0.16$, Chi-square test with Yates' correction). Cell polarity was evaluated at DPI 13. (e) Representative reconstructions of the same cell at 12 different time points (groups of 2 consecutive time points, DPI 13-45). Colors highlight pruned (orange) and added (green) dendrites for each group. Scale bar: $25 \mu \mathrm{m}$. (f-j) Box plots illustrating the normalized total dendritic branch length (f), numbers of dendrites (g), primary dendrites (h), branch points (i) and endings (j). For each cell, the data were normalized to the respective value measured at DPI $13(P<0.05$, Friedman test followed by Dunn's multiple comparisons test; $n=34$ cells, 8 mice). (k) Schematic diagram illustrating the Sholl analysis procedure. Scale bar: $30 \mu \mathrm{m}$. (I) Graph comparing the mean numbers of intersections with Sholl spheres for cells imaged at DPI 13 (red) and DPI 45 (black). Error bars: SEM. Grey zones in (k) and (I) indicate the regions where the numbers of intersections showed significant differences between the two age groups $\left({ }^{\star} P<0.05\right.$, Wilcoxon signed rank test; $n=34$ cells, 8 mice).

Figure 3. Dendritic morphology cannot predict the fate of adult-born JGNs. (a) Representative reconstructions of two eliminated (grey) adult-born JGNs and corresponding surviving (black) cells from the same mice at two days before death (DBD 2). Left: PGC-like cells, right: SAC-like cells. Scale bar: $25 \mu \mathrm{m}$. (b-f) Box plots showing the normalized total dendritic branch length (b), numbers of dendrites (c), primary 
dendrites (d), branch points (e) and endings (f) measured at DBD 3, DBD 2 and DBD 1. $(\mathbf{g}, \mathbf{h})$ Normalized fractions of pruned $(\mathbf{g})$ and added (h) dendritic endings at DBD 3-2 and DBD 2-1. Data obtained in subsequently eliminated cells were normalized to the respective median value obtained in corresponding surviving cells of the same type recorded in the same animal at the same time point $(P>0.05$, Wilcoxon signed rank test; $\mathrm{n}=10$ eliminated cells and 63 surviving cells, 6 mice).

Figure 4. The fate of adult-born JGNs is not determined by their odorresponsiveness. (a) A schematic diagram showing the experimental timeline. (b) MIP (63-135 $\mu \mathrm{m}$, step $3 \mu \mathrm{m}$ ) images representing stable (blue arrows), unstable (red arrows) and late-coming (orange arrow) Twitch-2B+ adult-born JGNs at DPI 18-22. Scale bar: 50 $\mu \mathrm{m}$. (c) Box plot showing the fraction of stable cells at DPI 18-22 ( $n=11$ mice). (d) Box plot showing the fractions of cells eliminated at DPI 22-44 in Twitch-2B+ cell and RGB+ cell groups ( $P=0.97$, Mann-Whitney test; $\mathrm{n}=11$ and 12 mice, respectively). (e) Representative image (average of 140 consecutive frames) of a Twitch-2B ${ }^{+}$adult-born JGN at DPI 24. Scale bar: $10 \mu \mathrm{m}$. (f) Traces illustrating changes in fluorescence intensity of mCerulean3 and $\mathrm{cpVenus}^{\mathrm{CD}}$ and the Twitch-2B ratio trace recorded from the somatic region of interest shown in (e) in response to a 4-s-long application of the mixture of 7 odorants (ethyl-acetate, butanal, pentanal, ethyltiglate, propanal, methylpropionate and ethyl-butyrate). (g) Box plot summarizing the fraction of odor responding cells at DPI 20-32 ( $\mathrm{n}=11$ mice). (h) Bar graphs showing the fractions of odor responding and odor non-responding cells among the eliminated (left) and surviving (right) cells $(P=0.74$, Chi-square test with Yates' correction). (i,j) Box plots showing the normalized maximum $\Delta \mathrm{R} / \mathrm{R}$ amplitude (i) $(P=0.49$, Wilcoxon signed rank test $)$ and area 
under the curve $(\mathrm{j})(P=0.23$, One sample $t$ test). The medians of subsequently eliminated cells were normalized to the medians of corresponding surviving cells recorded in the same animal at the same time point $(n=12$ odor responding eliminated and 112 odor responding surviving cells, 8 mice).

Figure 5. Different basal Twitch-2B ratios and endogenous activity patterns in eliminated and surviving adult-born JGNs. (a) Graph illustrating the endogenous activity of adult-born JGNs and parameters used for its analyses. AUC: area under the curve. Scale bar: 5 s. (b-g) Connected dot graphs summarizing the median values of basal Twitch-2B ratio ( ${ }^{*} P<0.05$, Wilcoxon signed rank test) (b), maximum Twitch-2B ratio $\left({ }^{\star} P<0.05\right.$, Wilcoxon signed rank test) $(\mathbf{c})$, maximum $\Delta \mathrm{R} / \mathrm{R}$ amplitude $(P>0.05$, Paired $t$ test) (d), fraction of time spent above 2.4 (i.e. in the active state; ${ }^{*} P<0.05$, Wilcoxon signed rank test) (e), the normalized area under the curve (AUC/second; ${ }^{*} P<$ 0.05 , Wilcoxon signed rank test) (f), fraction of time spent below 2.0 (in the non-active state; ${ }^{*} P<0.05$, Wilcoxon signed rank test) (g) of eliminated cells and corresponding surviving cells in the same mice recorded at DBD 6 (96-144 hours before cell death), DBD 4 (48-96 hours before cell death) and DBD 2 (0-48 hours before cell death). DBD 6: $n=14$ eliminated and 302 surviving cells from 7 mice; DBD 4: 20 eliminated and 466 surviving cells from 8 mice; DBD 2: 16 eliminated and 371 surviving cells from 8 mice.

\section{Supplementary Figure 1. 4D structural plasticity analysis of dendritic remodeling} in stable surviving adult-born JGNs. (a,b) Box plots showing the fractions of pruned (a) and added (b) dendritic endings in stable surviving adult-born JGNs $\left({ }^{\star} P<0.05\right.$, Oneway repeated measures ANOVA followed by Holm-Sidak's multiple comparisons test; $n$ 
$=34$ cells, 8 mice) .

\section{Supplementary Figure 2. Development and plasticity of the dendritic tree in stable} surviving adult-born PGCs. (a) Representative reconstructions of 4 adult-born PGCs at DPI 45. Scale bar: $25 \mu \mathrm{m}$. (b-f) Box plots summarizing the normalized total dendritic branch length (b) $\left({ }^{\star} P<0.05\right.$, One-way repeated measures ANOVA followed by HolmSidak's multiple comparisons test) as well as numbers of dendrites $(\mathbf{c})(P>0.05$, Oneway repeated measures ANOVA followed by Holm-Sidak's multiple comparisons test), primary dendrites $(\mathbf{d})\left({ }^{*} P<0.05\right.$, Friedman test followed by Dunn's multiple comparisons test), branch points $(\mathbf{e})\left({ }^{\star} P<0.05\right.$, One-way repeated measures ANOVA followed by Holm-Sidak's multiple comparisons test) and endings $(\mathbf{f})\left({ }^{*} P<0.05\right.$, One-way repeated measures ANOVA followed by Holm-Sidak's multiple comparisons test) in stable surviving adult-born PGCs. For each cell, the data were normalized to the respective values measured at DPI 13 ( $n=13$ PGCs, 7 mice). (g,h) 4D structural plasticity analysis showing the fractions of pruned $(\mathbf{g})$ and added (h) endings in stable surviving adult-born PGCs $\left({ }^{*} P<0.05\right.$, One-way repeated measures ANOVA followed by Holm-Sidak's multiple comparisons test; $n=13$ PGCs, 7 mice). (i) Sholl analyses of adult-born PGCs at DPI 13 (red) and DPI 45 (black) ( ${ }^{*} P<0.05$, Wilcoxon signed rank test, $\mathrm{n}=13 \mathrm{PGCs}, 7$ mice). Error bars: SEM.

\section{Supplementary Figure 3. Development and plasticity of the dendritic tree in stable} surviving adult-born SACs. (a) Representative reconstructions of 3 adult-born SACs at DPI 45. Scale bar: $50 \mu \mathrm{m}$. (b-f) Box plots illustrating the normalized total dendritic branch length (b) $\left({ }^{\star} P<0.05\right.$, One-way repeated measures followed by Holm-Sidak's 
multiple comparisons test) as well as numbers of dendrites $(\mathbf{c})(P>0.05$, Friedman test followed by Dunn's multiple comparisons test), primary dendrites (d) $\left({ }^{*} P<0.05\right.$, Friedman test followed by Dunn's multiple comparisons test), branch points $(\mathbf{e})(P>$ 0.05, Friedman test followed by Dunn's multiple comparisons test), and endings (f) $(P>$ 0.05, One-way repeated measures ANOVA followed by Holm-Sidak's multiple comparisons test) in stable surviving adult-born SACs. For each cell, the data were normalized to the respective values measured at DPI 13 ( $n=14$ SACs, 5 mice). (g,h) 4D structural plasticity analysis showing the fractions of pruned (g) and added (h) dendritic endings at DPI 13-45 ( ${ }^{\star} P<0.05$, Friedman test followed by Dunn's multiple comparisons test; $n=14$ SACs, 5 mice). (i) Sholl analyses of SACs at DPI 13 (red) and DPI 45 (black) ( $P<0.05$, Wilcoxon signed rank test; $\mathrm{n}=14$ SACs, 5 mice). Error bars: SEM.

\section{Supplementary Figure 4. An initial period of rapid dendritic growth in surviving} adult-born JGNs at DPI 9-13. (a) Representative reconstructions of 3 adult-born JGNs at DPI 9. Scale bar: $25 \mu \mathrm{m}$. (b-f) Box plots summarizing the normalized total dendritic branch length (b) as well as numbers of dendrites (c), primary dendrites (d), branch points (e) and endings (f) in surviving adult-born JGNs at DPI 9-13. For each cell, the data were normalized to the respective values measured at DPI 13 ( ${ }^{*}<0.05$ for all comparisons, Friedman test followed by Dunn's multiple comparisons test; $n=15$ JGNs, 4 mice). (g) Sholl analyses showing the number of dendritic intersections with Sholl spheres at DPI 9 (green) and DPI 13 (red) $\left({ }^{*} P<0.05\right.$, Wilcoxon signed rank test; $\mathrm{n}=15$ JGNs, 4 mice). Error bars: SEM. 
Supplementary Figure 5. Visualization of the death of Twitch-2B+ adult-born JGNs. (a) Images showing 1 eliminated (magenta arrow) and 1 surviving (green arrow) Twitch$2 \mathrm{~B}^{+}$adult-born JGNs in the same FOV at DPI 18 (left) and DPI 20 (right). Each image is an average of 830 consecutive frames. Scale bar: $20 \mu \mathrm{m}$. (b) Images showing 1 eliminated (magenta arrow) and 4 surviving (green arrows) cells in the same FOV at 8 different time points. DPI 34 was defined as the day of death. Each image is an average of 830 consecutive frames. Scale bar: $20 \mu \mathrm{m}$.

\section{Supplementary Figure 6. Stability of basal Twitch-2B ratios and endogenous} activity patterns in eliminated and surviving adult-born JGNs. (a-f) Graphs showing the differences between DBD 6, DBD 4 or DBD 2 and DBD 6 for the following parameters: basal Twitch-2B ratio (a), maximum Twitch-2B ratio (b), maximum $\Delta R / R$ amplitude (c), fraction of time spent above 2.4 (d), normalized AUC (AUC/second) (e), fraction of time spent below 2.0 (f). Different symbols correspond to different cell groups as indicated in (c) $(P>0.05$ for all comparisons, Friedman test for comparison of the same groups at different time points, Wilcoxon signed rank test for comparison of different groups at the same time points; $n=8$ eliminated cells and 138 surviving cells, 5 mice).

Supplementary Figure 7. Analysis of $\left[\mathrm{Ca}^{2+}\right]_{i}$ fluctuations in adult-born JGNs. (a, b) Representative traces obtained from adult-born JGNs with fluctuating (a) and nonfluctuating (b) Twitch-2B ratios. Scale bar: 10 s. (c-e) Bar graphs summarizing the fractions of cells with fluctuating and non-fluctuating Twitch-2B ratios among the 
subsequently eliminated and surviving adult-born JGNs at DBD 6 (c), DBD 4 (d) and DBD $2(\mathbf{e})(P>0.05$ for all comparisons, Chi-square test with Yates' correction).

Supplementary Figure 8. Impact of the improved image quality on the dendritic complexity of adult-born JGNs. (a) Representative MIP images (14-52 $\mu \mathrm{m}$, step $2 \mu \mathrm{m}$ ) and 3D reconstructions of the same adult-born JGN imaged under high (left) and low (right) noise conditions. Scale bar: $20 \mu \mathrm{m}$. (b) Boxplots showing the normalized signalto-background (left) and signal-to-noise (right) ratios ( ${ }^{*} P<0.001$, Wilcoxon signed rank test, $n=12$ ). For each cell, the SBR (or SNR) in the low-noise group was normalized to the respective SBR (or SNR) in the high-noise group. (c) Boxplots summarizing the normalized TDBL as well as number of dendrites, primary dendrites, branch points and endings ( ${ }^{*} P<0.001$, Wilcoxon signed rank test, $\mathrm{n}=12$ ). For each cell, the data in the low-noise group were normalized to the respective values in the high-noise group. 
Figure 1. Longitudinal mapping of adult-born JGN's fate

a

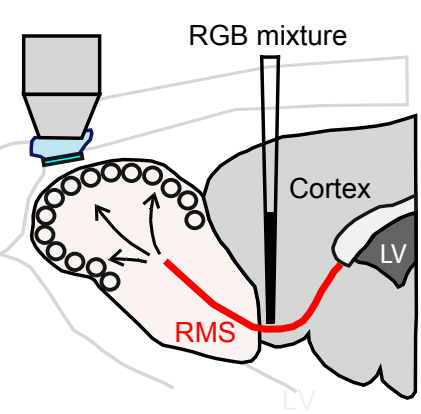

C
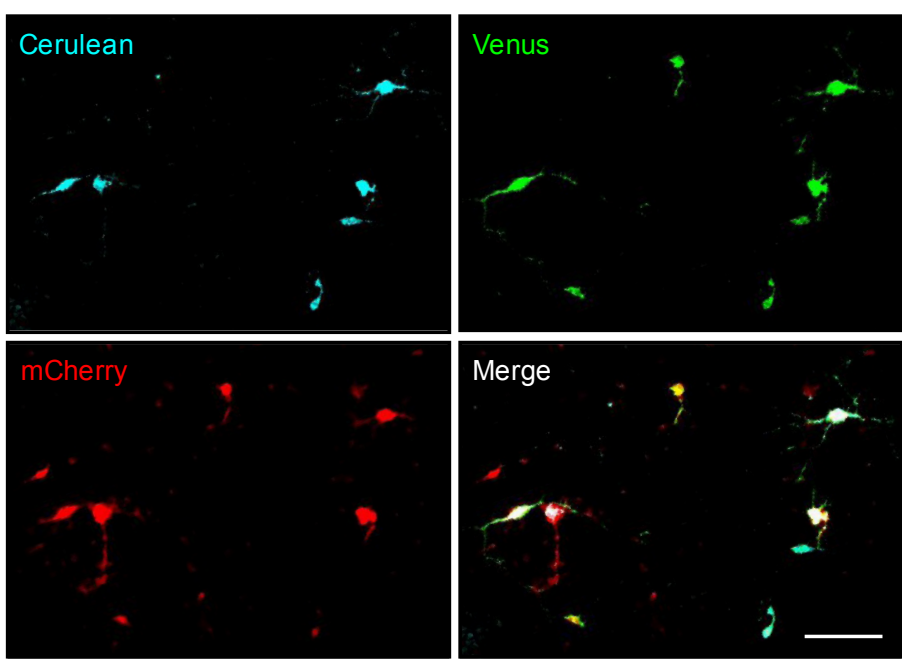

f $\quad$ DPI 12-45

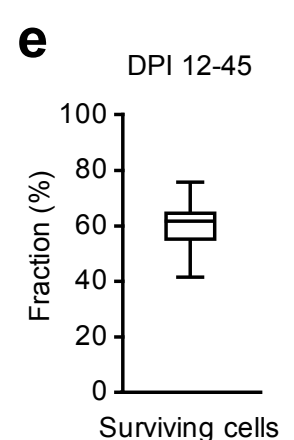

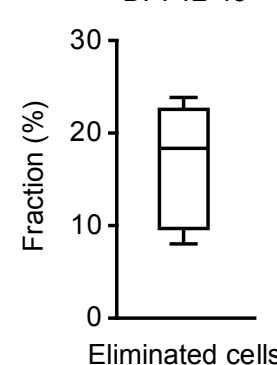

b

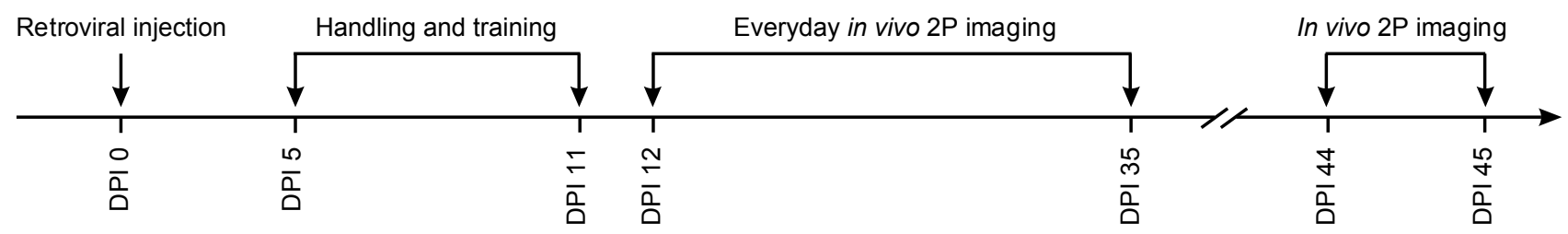

d

$800 \mathrm{~nm}$ excitation wavelength

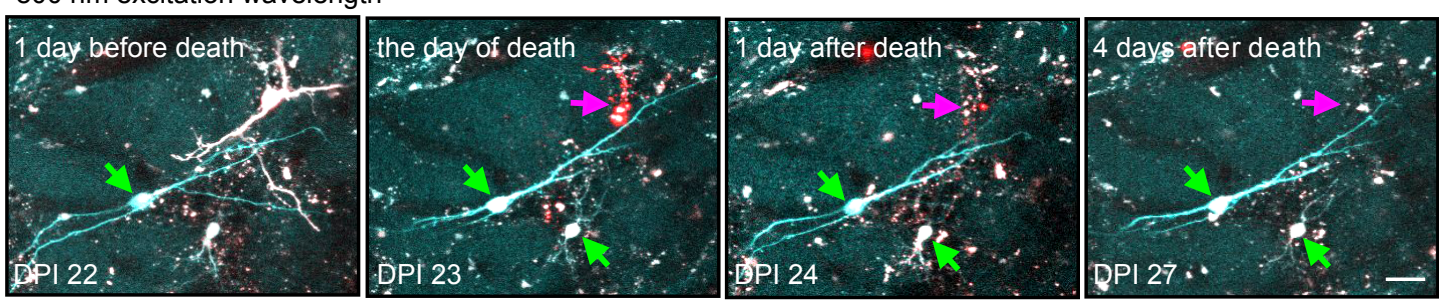

$900 \mathrm{~nm}$ excitation wavelength

A Surviving

4 Eliminated

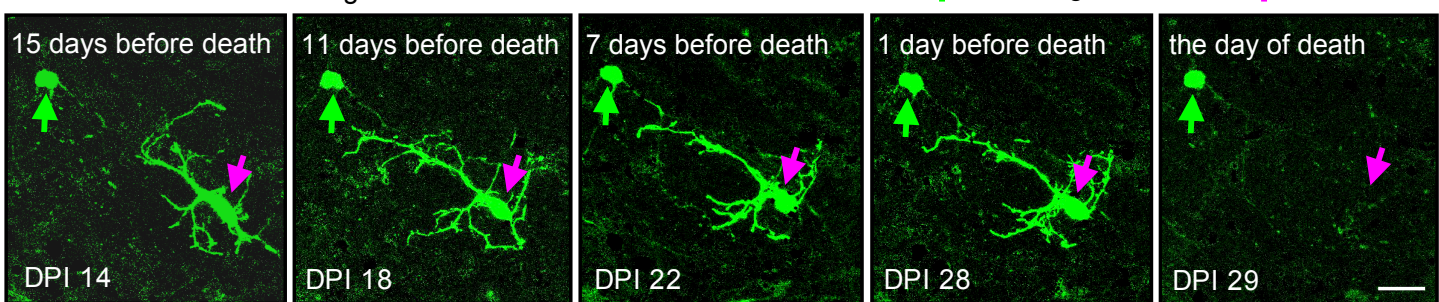

h Cumulative distribution of eliminated cells i Cumulative distribution of uncertain cells
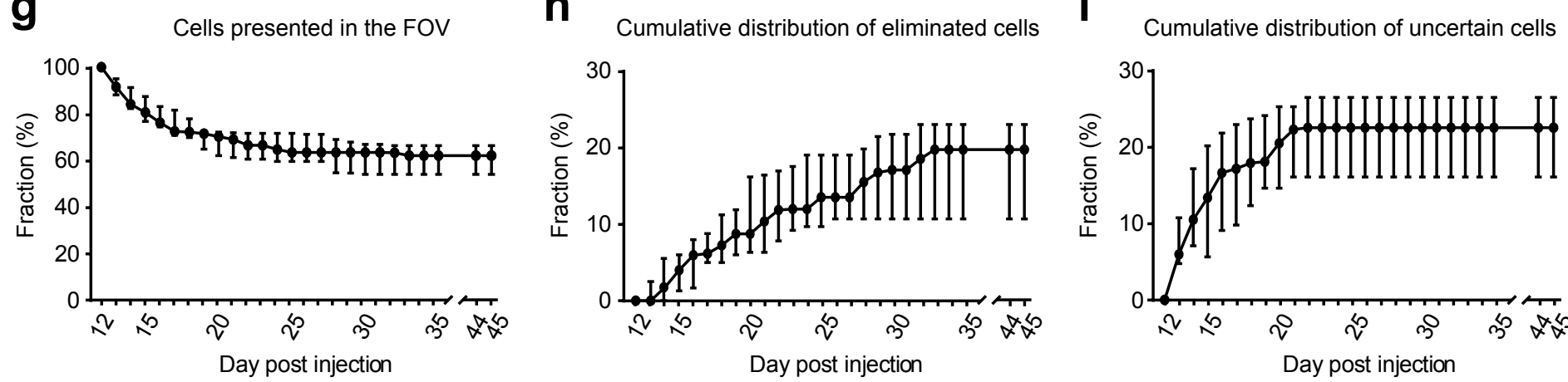


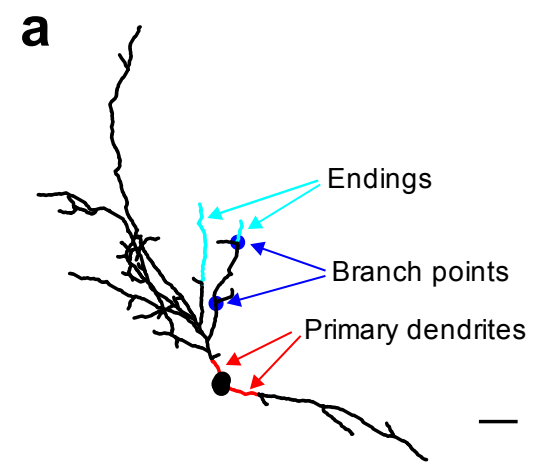

DPI 13
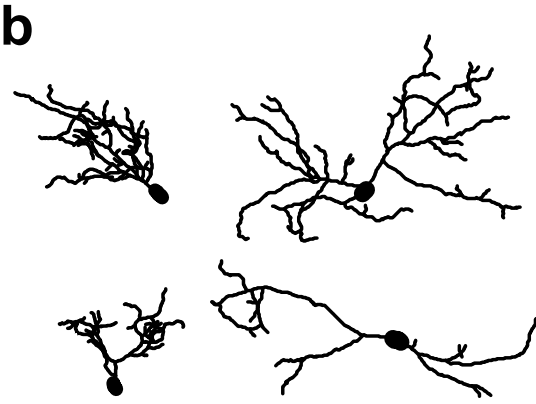

Unipolar

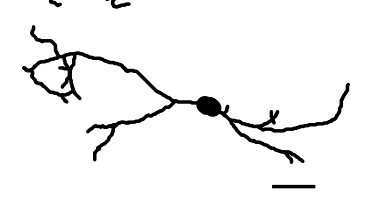

Multipolar
C

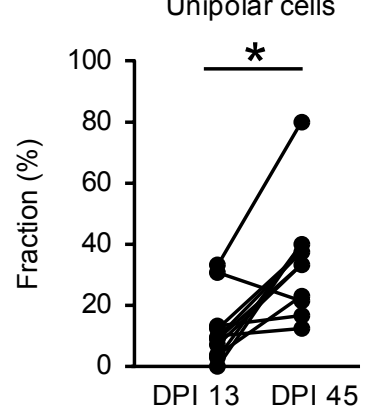

d

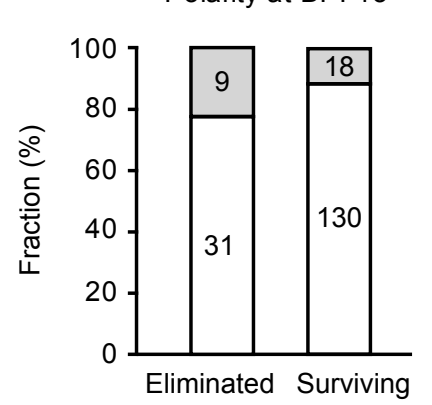

$\square$ Unipolar $\square$ Multipolar

e
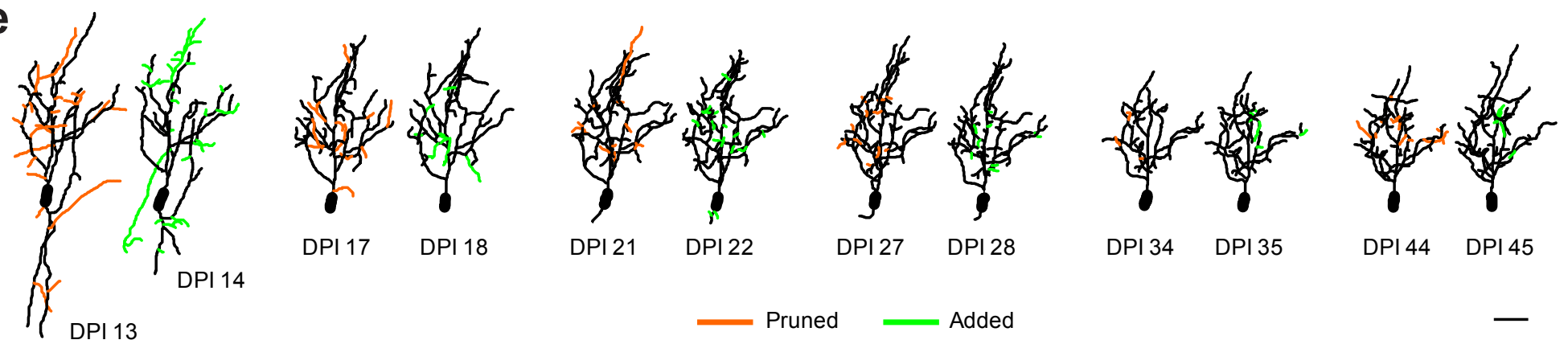

DPI 17

DPI 18

DPI 21

DPI 22

DPI 27 DPI 28

DPI 34 DPI 35

DPI 44

DPI 45

- Pruned

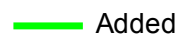

f

Total dendritic branch length
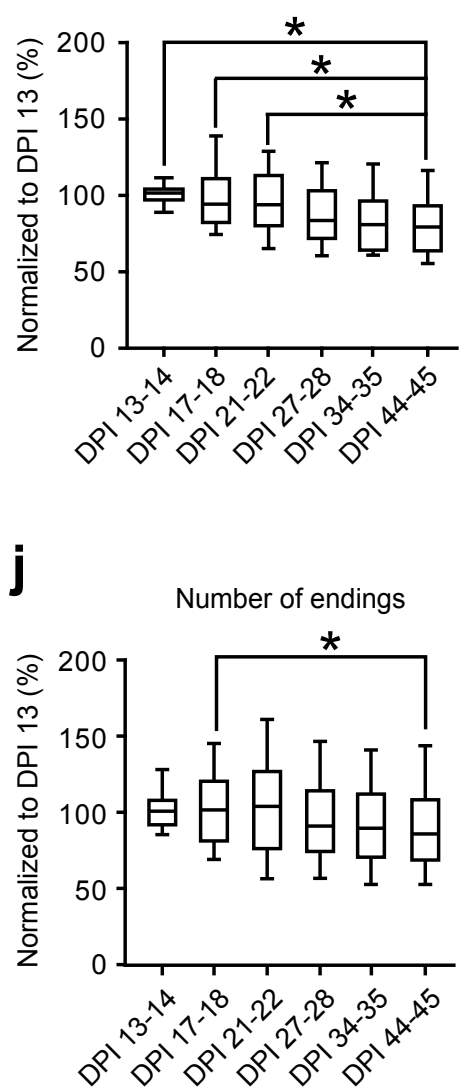

g

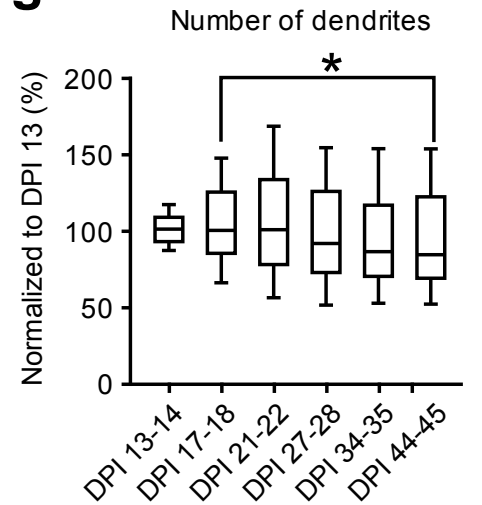

k

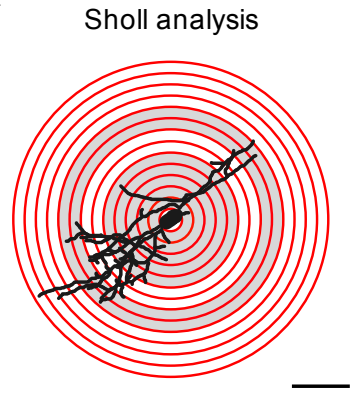

h Number of primary dendrites
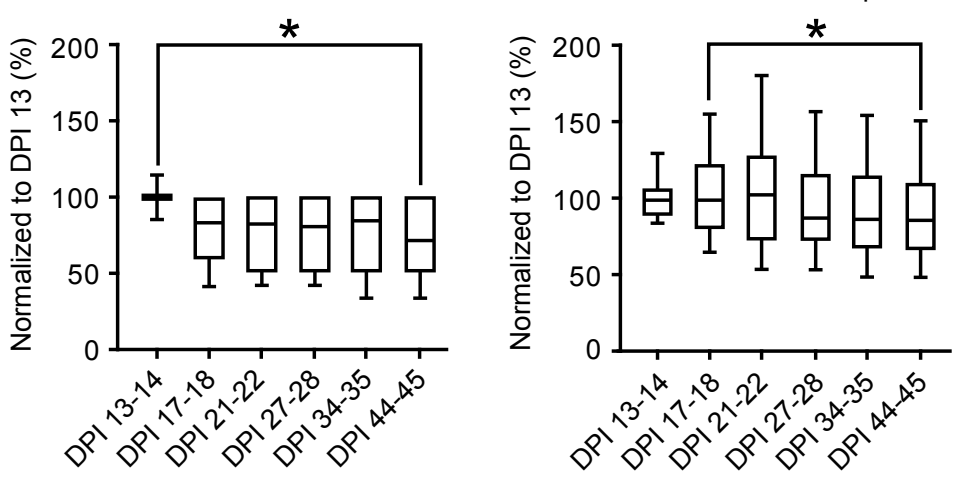

I

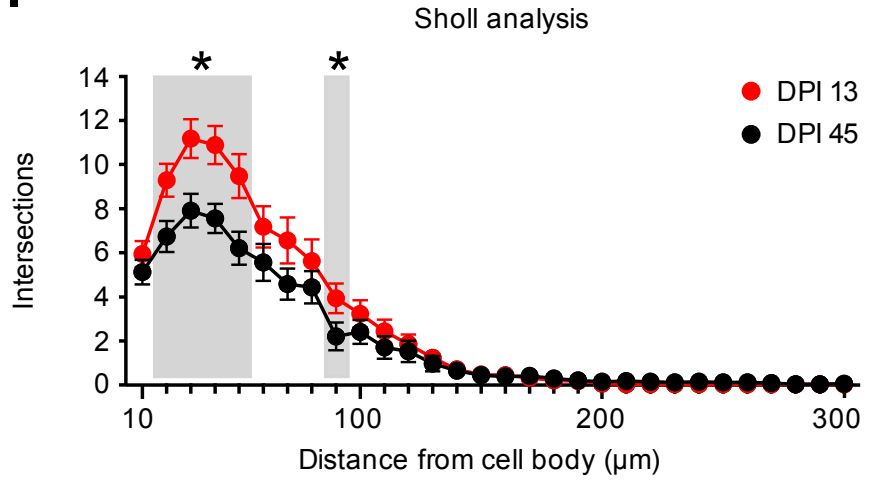


Figure 3. Dendritic morphology cannot predict the fate of adult-born JGNs

a

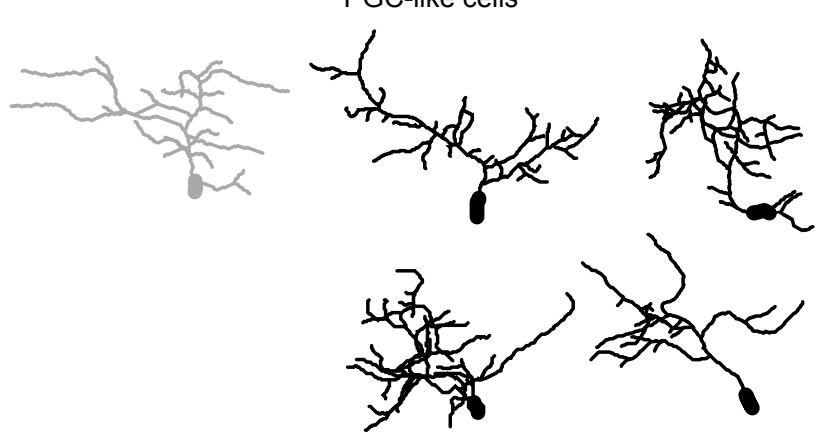

Eliminated
- Surviving

C

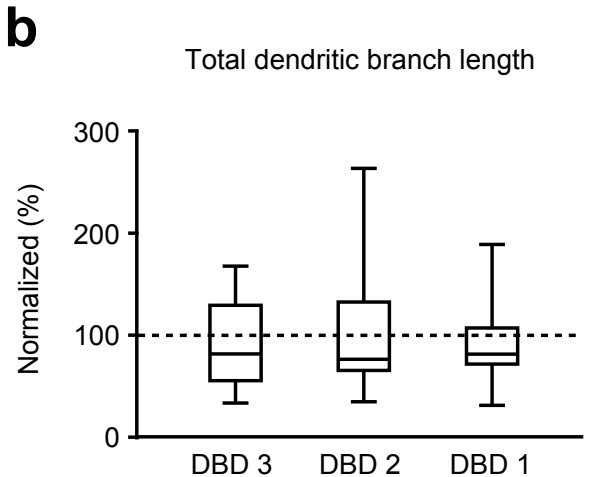

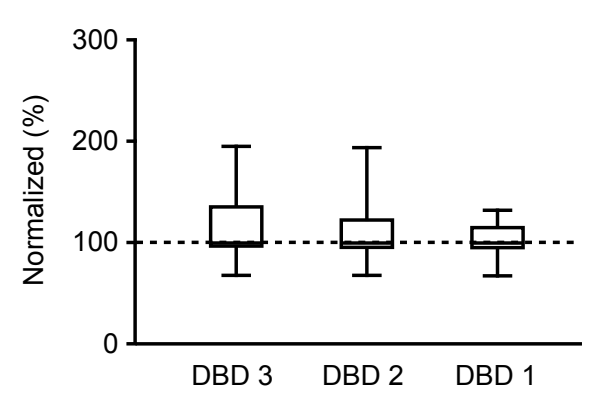

d

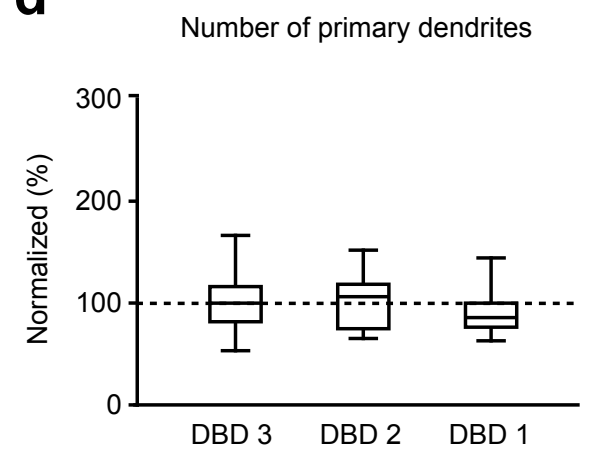

SAC-like cells

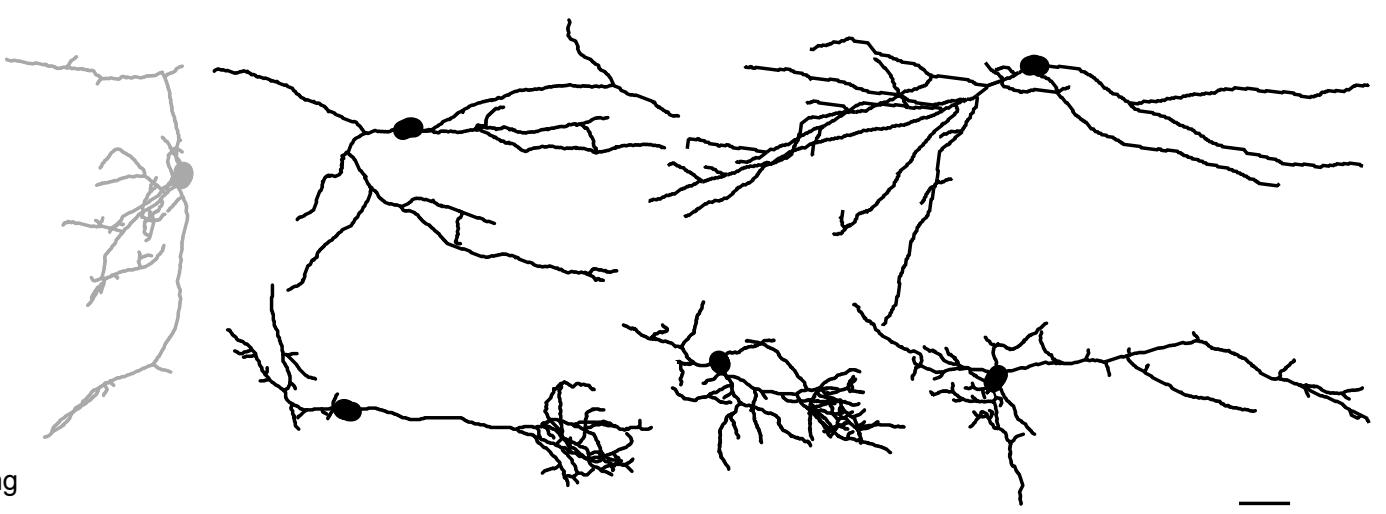

e

Number of branch points

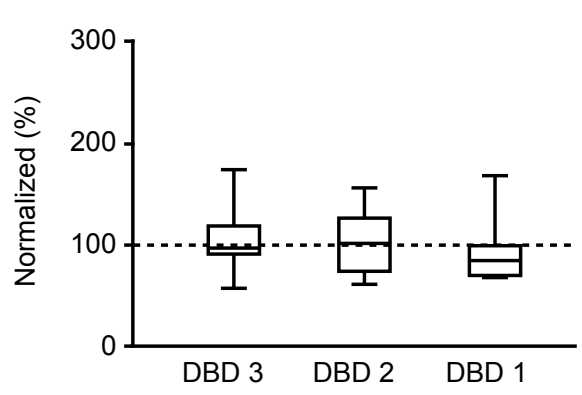

f

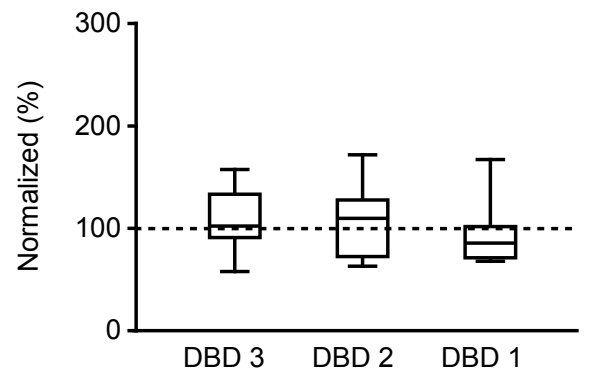

g

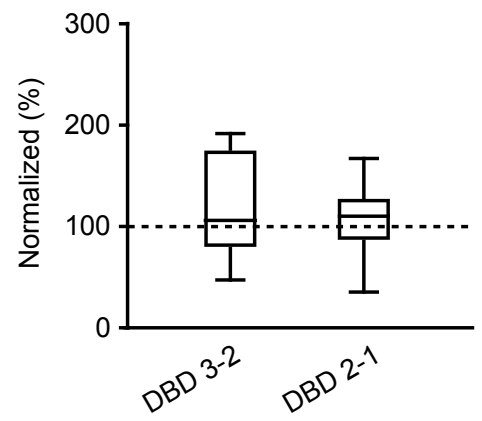

h

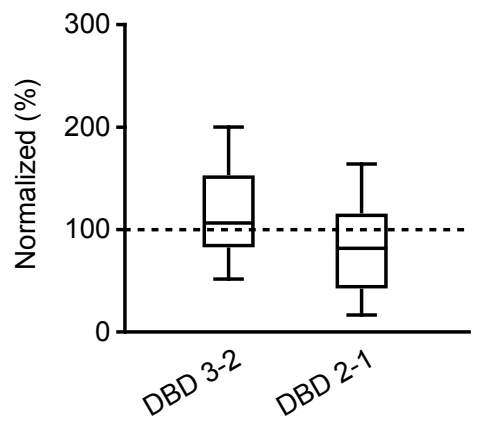


bioRxiv preprint doi: https://doi.org/10.1101/2020.09.09.286591; this version posted September 9, 2020. The copyright holder for this preprint (which was not certified by peer review) is the author/funder. All rights reserved. No reuse allowed without permission.

Figure 4. Fate of adult-born JGNs is not determined by their odor-responsiveness

a

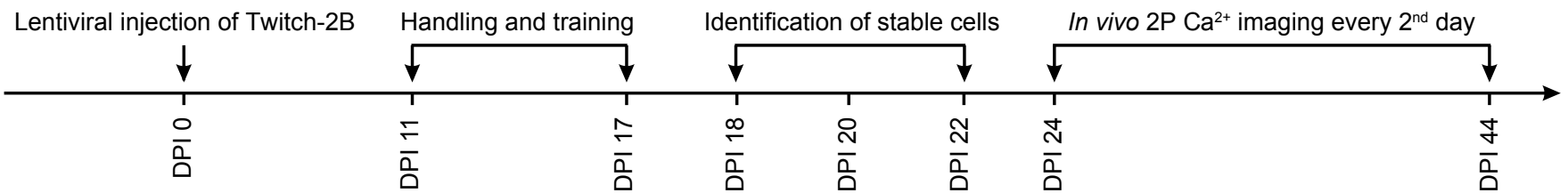

b

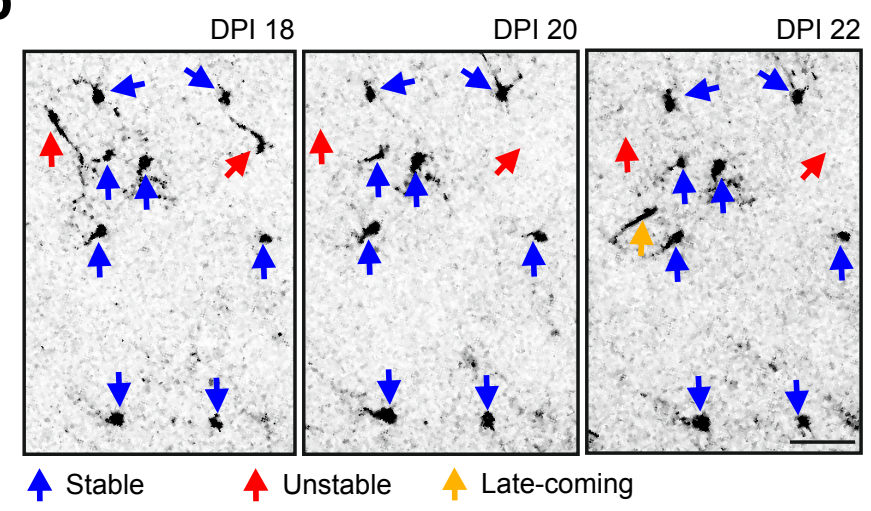

C

DPI 18-22

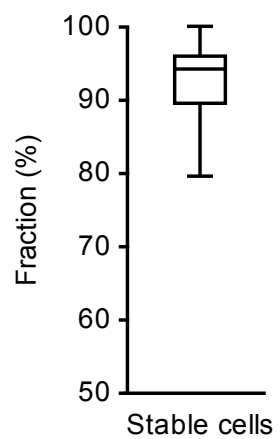

d

Eliminated cells at DPI 22-44

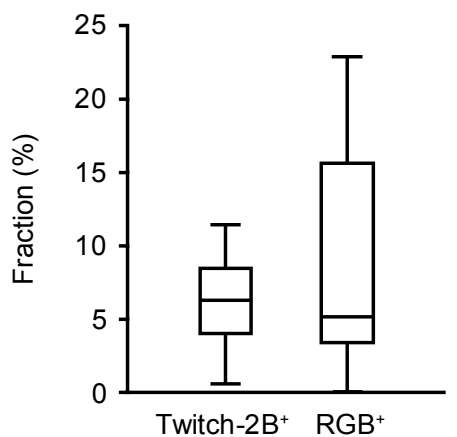

g

DPI 20-32

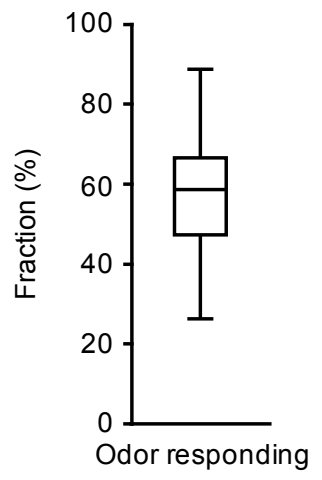

h

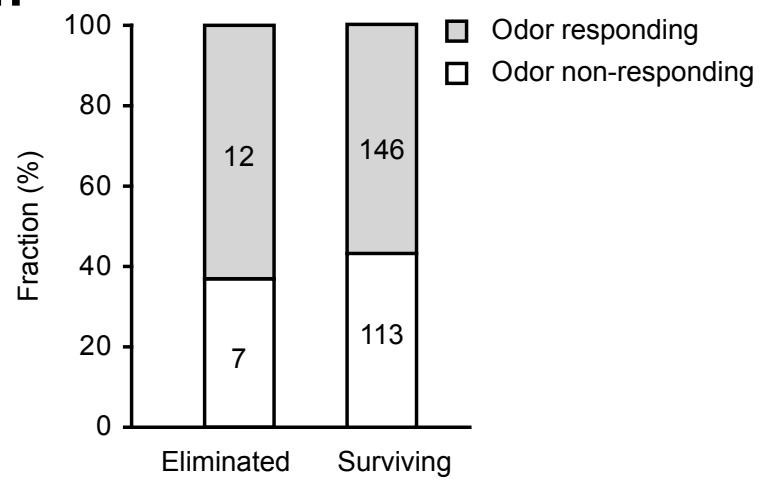

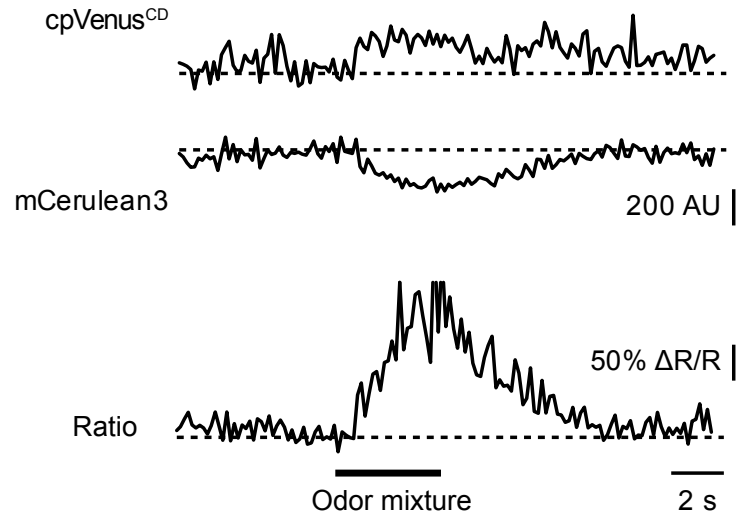

i

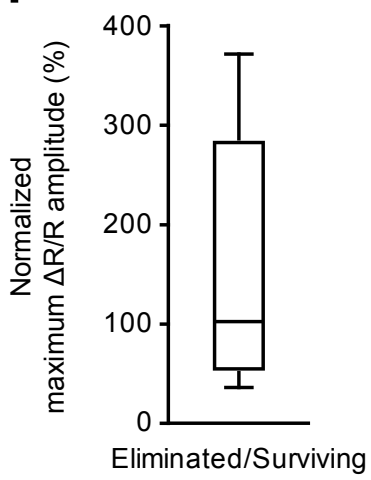

j

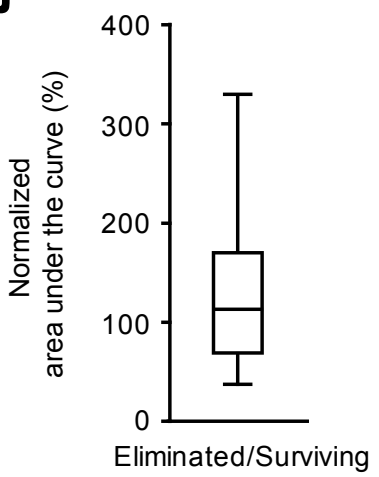




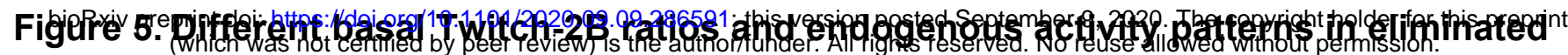
and surviving adult-born JGNs

$a$
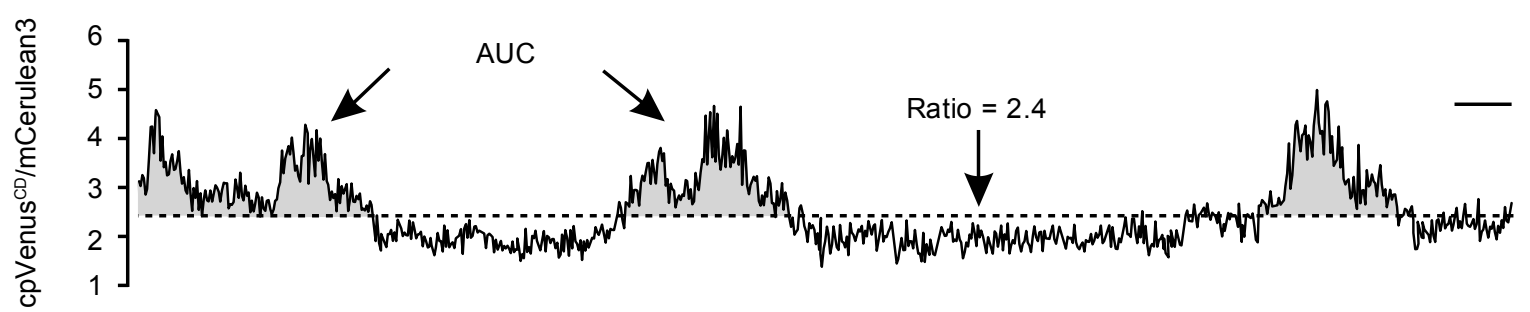

b

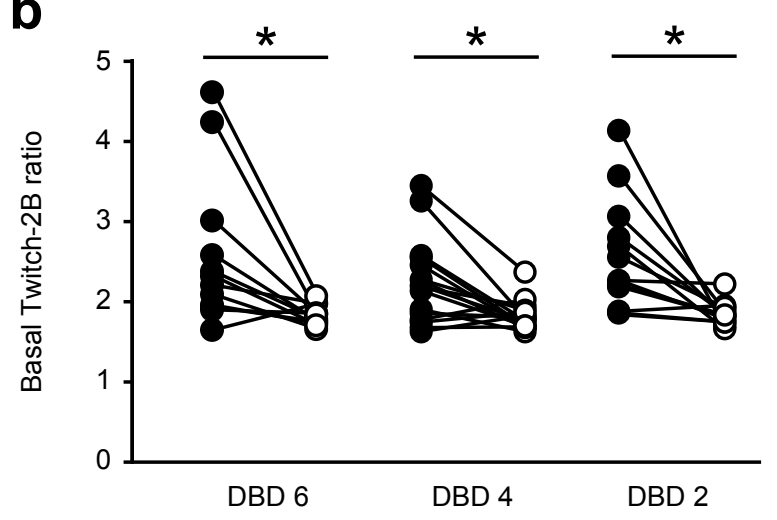

d

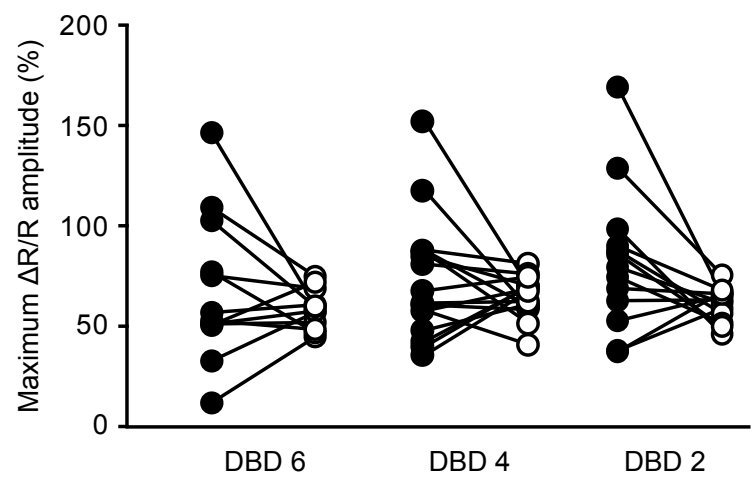

$\mathbf{f}$

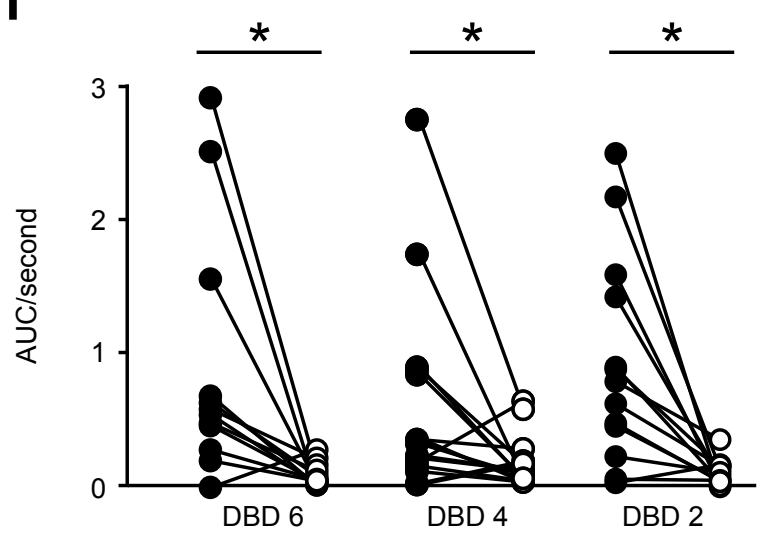

Eliminated adult-born JGNs

O Surviving adult-born JGNs
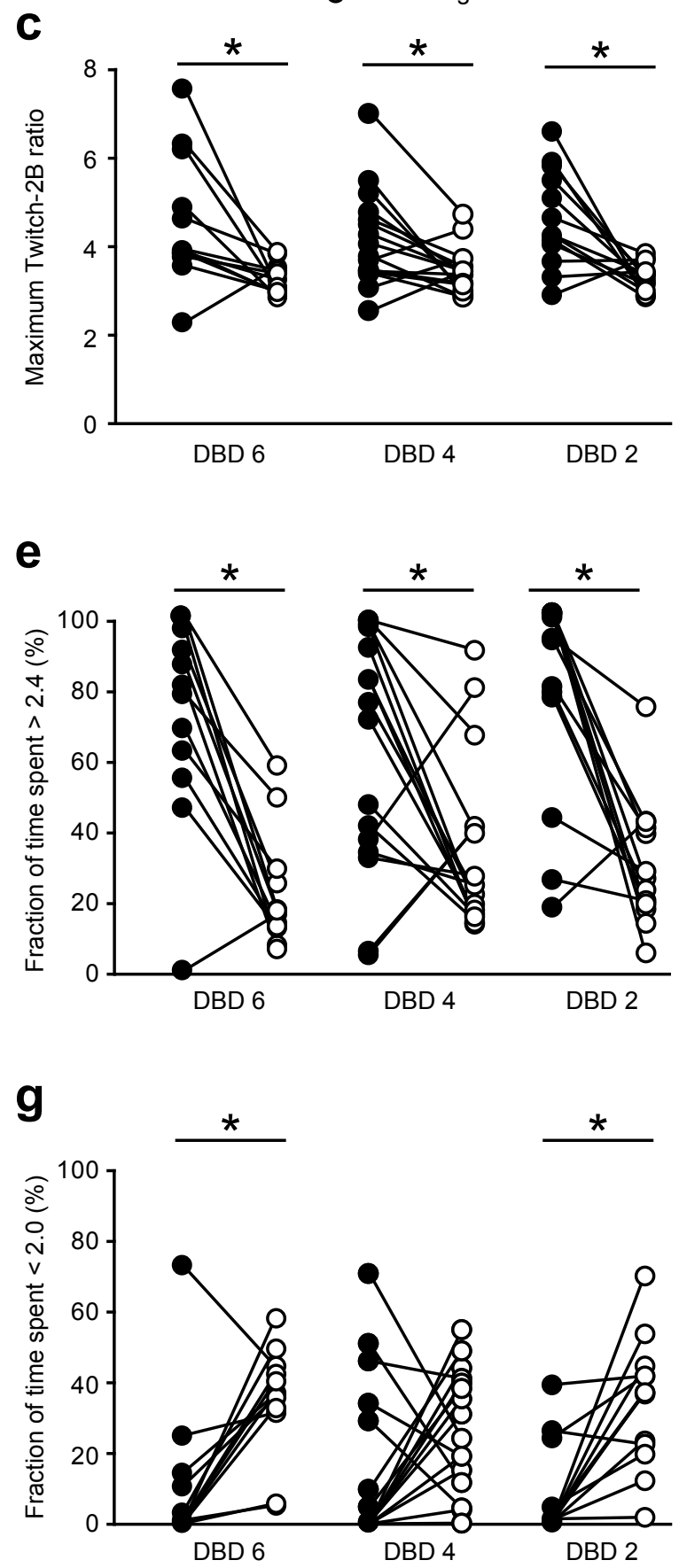